\title{
L'expérience professionnelle : expériences sédimentées et expériences épisodiques
}

Professional experience: sedimented experiences and episodic experiences

Janine Rogalski et Jacques Leplat

\section{(2) OpenEdition}

Journals

Édition électronique

URL : http://journals.openedition.org/activites/2556

DOI : 10.4000/activites.2556

ISSN : $1765-2723$

Éditeur

ARPACT - Association Recherches et Pratiques sur les ACTivités

Référence électronique

Janine Rogalski et Jacques Leplat, «L'expérience professionnelle : expériences sédimentées et expériences épisodiques », Activités [En ligne], 8-2 | octobre 2011, mis en ligne le 15 octobre 2011, consulté le 30 avril 2019. URL : http://journals.openedition.org/activites/2556 ; DOI : 10.4000/ activites. 2556

\section{(c) (i) (9)}

Activités est mis à disposition selon les termes de la licence Creative Commons Attribution - Pas d'Utilisation Commerciale - Pas de Modification 4.0 International. 


\title{
L'expérience professionnelle : expériences sédimentées et expériences épisodiques
}

\author{
Janine Rogalski \\ Directeur de recherche honoraire CNRS, laboratoire CHArt Université Paris8, 2 rue de la liberté, 93526 Saint-Denis \\ Cedex, France; rogalskij@univ-paris8.fr
}

\section{Jacques Leplat}

Directeur honoraire EPHE, CRDT, CNAM, 41 rue Gay-Lussac, 75005 Paris, France; jacques.leplat@wanadoo.fr

\begin{abstract}
Professional experience: sedimented experiences and episodic experiences. Professional experience is defined as the product of practice resulting from tasks performed in a given domain of work, over a relatively long period. We put forward the idea that professional experience covers two modalities: "sedimented" experiences are centred on the repetition of tasks in all their variability; "episodic" experiences are centred on the singularity of the situations encountered, from anomalous events to paradigmatic cases. We discuss how these modalities are articulated in relation to the varying demands of the cognitive operations involved in professional tasks (memory, categorisation, analogical reasoning, decision making...). Analysis of experience depending on its "sedimented" and "episodic" modalities is used to question how they contribute to the development of competencies, discuss what may go wrong with the experience, clarify the conditions required for acquiring competencies and draw some conclusions that may prove useful in devising training programmes.
\end{abstract}

KEYWORDS

Professional experience, sedimented experiences, episodic experiences, development, professional competence.

\section{1.- Introduction}

La notion d'expérience a de multiples significations comme le révèlent des expressions du langage courant : acquérir ou avoir une expérience, avoir fait une expérience (du danger, de la souffrance...). On parle aussi de l'expérience d'un opérateur, d'un savoir d'expérience, etc. Cette multiplicité, explicitée par exemple par Courtois (1989, pp. 7-12), apparaît encore élargie dans l'analyse que développe Mayen (2008) sur la problématique de la validation des acquis de l'expérience, où il rappelle que ces significations « dessinent des configurations conceptuelles différentes » (Mayen \& Mayeux, 2003).

En fait, ce terme polysémique peut renvoyer au moins à trois significations pertinentes du point de vue de la formation et du développement de la compétence professionnelle : 1) l'expérience peut être conçue comme issue de l'activité matérielle ou symbolique;2) elle peut être relative à l'ensemble des connaissances et habiletés acquises par ces voies au cours d'une certaine période; 3 ) elle peut être conçue comme événement ou occurrence qui laisse une impression sur quelqu'un (il s'y ajoute par ailleurs l'expérience scientifique, dont nous ne parlerons pas).

\section{- Expérience et formation}

On retiendra ici que l'expérience désigne ce que le sujet acquiert par la pratique, c'est-à-dire par l'exécution de tâches d'un domaine professionnel, sur un temps plus ou moins long.

Le domaine de l'expérience est à distinguer de celui des connaissances acquises par une formation systématique et aussi par diverses voies hors de l'exécution proprement dite du travail (consultation 
de documents, activités hors travail, etc.). L'action régie par l'expérience est d'abord conduite par les données du monde de l'action (data-driven processing) : elle est ainsi de type réactif (Norman, 1993, p. 24). L'expérience, outre qu'elle est toujours située (et d'abord contextualisée), met en jeu d'autres dimensions que cognitives, et dépasse nécessairement en cela les connaissances acquises en formation. Pour Fischer (2002), «L'expérience est fondée sur la perception sensorielle, mais elle n'est pas confinée à cela. L'expérience porte les empreintes de toute la personne. Elle contient des idées et des concepts, des pensées et des émotions et elle ne se confond donc pas avec la perception immédiate » (p. 130).

On trouvera des commentaires historiques sur l'expérience, au sens qui nous intéresse ici, dans le livre de Courtois et Pineau (1991) consacré à la « Formation expérientielle des adultes ». La formation expérientielle y est définie « comme une formation par contact direct, mais réfléchi. Par contact direct, c'est-à-dire sans médiation de formateurs, de programme, de livre, d'écran et même de mots...» (p. 29) (point que nous serons amenés à discuter).

\section{- Expérience et compétence}

Nous parlons dans ce texte de compétence et non d'expertise pour éviter les ambiguïtés liées aux acceptions diverses du terme « expertise ». En fait dans la littérature sur « l'expertise » apparaissent deux notions : une «notion forte d'expertise [qui] est celle d'excellence dans un domaine : un expert est celui qui maîtrise le mieux les situations, qui fait montre d'une meilleure performance [...] ; l'autre notion étant celle de la professionnalité établie », qui se manifeste avec différents niveaux de compétence (Rogalski \& Marquié, 2004, pp. 149-150). Dans une revue des recherches sur l'expertise, la problématique de l'expérience est traitée en termes de "reiterate practice" (Charness, Tuffish, Krampe, Reingold, \& Vysukova, 2005 ; Ericsson, 2005 ; Rikers \& Paas, 2005). Il n’y est pas introduit de distinction entre des modalités de l'expérience, pourtant en jeu dans ce domaine'.

Distinguer expérience et connaissances apprises, deux déterminants de la compétence professionnelle, ce n'est pas les opposer, car elles peuvent se développer conjointement : les connaissances peuvent faciliter l'acquisition de l'expérience, et à travers l'expérience peuvent s'élaborer des connaissances explicitables. Cependant, alors que les connaissances de la formation visent le générique - qu'il s'agisse de concepts ou de procédures, l'expérience est liée à une situation : pour s'exprimer comme compétence, elle requiert souvent des conditions externes particulières, un environnement humain et technique bien défini. Si celui-ci est modifié, la compétence peut être dégradée. Ainsi, des transformations du dispositif peuvent plus ou moins invalider l'expérience comme le montre l'exemple souvent cité de l'ouvrier dont l'arrangement du poste de travail avait été " aménagé » (Linhart, 1978). Comme le dit Brassac (2008), « ... l'expérience ne se situe pas que dans l'encéphale d'un seul individu, mais dans un réseau complexe de ressources (allant du cerveau à la documentation et des collègues à la machine)» (p. 13). Dans une approche dite Work Process Knowledge - WPK (Boreham, Samurçay \& Fischer, 2002; Fischer, Boreham, \& Nyham, 2004), ce caractère situé de l'expérience est analysé en détail par Fischer $(2002 ; 2005)$ à partir de travaux développés dans le domaine manufacturier. Il y montre que la connaissance du processus de travail (WPK) comporte nécessairement des éléments propres au travail dans une entreprise donnée, où même « chaque machine a ses marottes! » (Fischer \& Rauner, 2002, p. 163).

Ces rappels montrent que l'expérience est une notion complexe qui peut s'envisager sous des angles divers : on a choisi ici l'un d'entre eux en défendant l'idée que cette notion présente deux modalités. On propose de les appeler respectivement « expériences sédimentées » et « expériences épisodiques ». La première modalité met l'accent sur la répétition des tâches au cours de la pratique, la

1. Par exemple, pour les échecs, il existe des expériences singulières de parties jouées ou analysées qui se distinguent de l'accumulation de résolution de multiples problèmes classiques de fin de partie. Par ailleurs, Ericsson relève des biais de diagnostic dans la pratique médicale liés aux expériences épisodiques: cas de routine plus disponibles que la connaissance des cas rares faisant obstacle à l'élaboration d'un diagnostic et d'un traitement appropriés. 
deuxième sur la singularité des situations rencontrées. Ces deux modalités sont à mettre en relation avec une distinction présente en psychologie cognitive dans le champ de la catégorisation entre type et occurrence, et par ailleurs entre mémoire "sémantique » et «mémoire épisodique ». Les expériences « sédimentées » renvoyant à une catégorisation en types de situations (qui sont mémorisées de manière "sémantique »), les expériences « épisodiques » à des situations singulières qui sont contextualisées, liées au hic et nunc de leur occurrence dans le travail (et objet d'une mémoire « épisodique $\gg$ ).

\section{Remarques}

Pour orienter la lecture de cet article, nous voudrions compléter cette introduction par quelques remarques. Nous nous sommes situés dans une perspective de psychologie ergonomique et notre référence est celle des situations professionnelles : il s'agit donc essentiellement ici de l'expérience acquise au cours du travail. Cette expérience porte sur des tâches de différentes « granularités » et des compétences à des niveaux de fonctionnement variés. Il peut s'agir d'opérations élémentaires très fréquemment réalisées et des « habiletés » qui leur sont liées (les «skills » en jeu dans la plupart des recherches expérimentales). À l'autre extrême, il peut s'agir de tâches se déployant dans la longue durée (de plusieurs heures à plusieurs mois, voire années pour certains projets de conception).

Nous ne cherchons pas ici à élaborer ou discuter une théorie générale de l'apprentissage et nous ne développerons pas cette question de granularité. Soulignons seulement que notre problématique déborde largement celle des tâches expérimentales sur lesquelles ont été élaborés des modèles de l'apprentissage de « compétences cognitives » (cognitive skills). La littérature classique sur ce sujet (qui sera évoquée à l'occasion) ne manque pas depuis le livre collectif dirigé par Anderson (1981), qui constitue encore aujourd'hui plus qu'une initiation au domaine. Ce sont généralement des tâches simples, bien définies et circonscrites par l'expérimentateur et le questionnement y porte sur l'effet de la pratique réitérée de telles tâches, dans des durées sans commune mesure avec celles relatives à la constitution de l'expérience professionnelle.

Les recherches sur les acquisitions de compétences professionnelles et la formation ont construit des modèles plus appropriés aux systèmes complexes que sont les situations de travail. Ces modèles sont centrés sur les contenus du domaine de tâches, avec des hiérarchies de connaissances opérationnelles. Le modèle général d'Anderson (ACT*, Anderson, 1982; puis ACT-R, Anderson, 1993) n'y est pas la référence centrale. Ainsi, dans un ouvrage collectif, Olson et Rasmussen (1989, p. 17) soulignent que «la compilation [centrale dans le modèle ACT] n'est pas une métaphore adaptée » pour rendre compte de l'acquisition de la connaissance procédurale dans l'expertise professionnelle. Moins radicalement sans doute, Salas et Rosen (2010, pp. 101 sq.) citent bien les facteurs mis en avant dans le modèle d'Anderson : "practice, automaticity and skilled performance », mais ils soulignent que les professionnels « qui ont atteint des niveaux experts de performance pour un domaine de tâches ont développé des mécanismes spécialisés [...] adaptés à la tâche ». Le modèle d'expertise qu'ils proposent comprend « un changement dans la perception et la compréhension d'une situation d'une perception fragmentée » vers une perception «plus holistique» (p. 103). La place des processus réflexifs y est aussi relevée. On peut considérer que ce modèle rejoint l'approche Work Process Knowledge.

Nos analyses des modalités de l'expérience professionnelle s'intègrent dans le cadre théorique de la didactique professionnelle élaboré à partir de Pastré (1997), qui constitue une approche alternative à la théorie de la cognition comme traitement de l'information et à la cognition située (Rogalski, 2004). Dans la ligne des prolongements proposés par Vergnaud $(1990$; 2001) à la théorie piagétienne (Rogalski, 2007), on souligne la place de la conceptualisation et de la construction de schèmes d'action dans le développement des compétences professionnelles².

Nous présentons ici les deux modalités que nous différencions dans l'expérience professionnelle,

2. Weill-Fassina et Pastré (2004) ont montré l'articulation de la psychologie ergonomique et de la didactique professionnelle pour aborer la question des compétences professionnelles. 
en relevant d'abord leur spécificité, puis leur articulation dans l'expérience produite chez le sujet et leurs apports au développement de la compétence professionnelle. La discussion de leur "productivité » et de leurs « pathologies » - au sens des limites, biais et impacts négatifs possibles - conduira à envisager brièvement les conditions du développement aussi bien du côté de l'activité du sujet que des interventions de nature didactique et de la conception de formation.

\section{2.- Les expériences sédimentées}

Les expériences sédimentées sont celles qui résultent de la répétition dans l'exécution d'une tâche. Le terme « sédimenté » n'est peut-être pas le meilleur en tant qu'il suggère l'idée d'empilement alors qu'il ne s'agit pas d'un simple empilement de traitement de situations particulières, mais plutôt d'une réorganisation, une restructuration. La sédimentation renvoie à un processus dynamique, par lequel l'expérience à un moment donné prend en compte l'expérience acquise au moment précédent en même temps que l'activité qui vient juste d'être réalisée. La nouvelle expérience est ainsi dépendante de la précédente en même temps qu'elle l'adapte d'une manière plus ou moins profonde. En l'absence de cette transformation, l'expérience se fige et n'est que la répétition de l'identique qui conduit à des fonctionnements purement de routine, à la sclérose.

Cette notion d'expérience sédimentée peut s'enrichir de sa confrontation avec celle d'habitus telle que l'a présentée et exploitée Bourdieu dans son ouvrage de 1980. L'habitus y est conçu comme une « histoire incorporée » : « produit de pratiques individuelles et collectives (...), il assure la présence active des expériences passées... » (p.91). Il est « la présence agissante de tout un passé dont il est le produit : il est ce qui confère aux pratiques une indépendance relative par rapport aux déterminations du présent immédiat » (p.94). Bourdieu souligne son caractère de structure structurée - prédisposée à fonctionner - et de structure structurante génératrice et organisatrice de pratiques » (p. 88). Ces propriétés, parmi d'autres, en font un concept voisin de celui qui est l'objet du texte présent.

On examinera dans cette partie le rôle des conditions qui interviennent dans le développement de l'expérience sédimentée : la répétition, la variabilité des conditions externes et de l'action de l'opérateur et le contexte non explicité.

\section{1.- Répétition et ajustement}

La répétition est une composante essentielle de la pratique, souvent la seule retenue et elle constitue un facteur majeur du développement de l'expérience. On a pu parler de la « loi omniprésente (ubiquitous) de la pratique » (Newell et Rosenbloom, 1981, p. 3), entendant par là « qu'elle se retrouvait dans les tâches les plus diverses, perceptivo-motrices, motrices, de résolution de problème, etc. » (p. 29). On peut dire d'elle ce que Norman (1993) dit de la pratique : «Qu'est-ce que la pratique apporte? Elle ajuste l'habileté, met en forme les structures de connaissances par des milliers de petites manières de telle façon que l'habileté qui, dans les premiers stades exigeait une pensée consciente et réfléchie, puisse ensuite être exécutée automatiquement sur le mode subconscient et expérientiel. La pensée (thought) expérientielle est une pensée ajustée (tuned)» (p. 29). C'est nous qui soulignons ce qui renvoie au processus de sédimentation.

À la suite de Gruber (1976-1977), Falzon et Teiger (1995) parlent de la « fonction constructive de la répétition ». Cette fonction résulterait du fait que la répétition permet de tirer parti « des variations de l'environnement pour l'élaboration de schèmes adaptatifs » (p. 4), ce que nous développons dans la partie suivante. La constitution de tels schèmes est un des composants de la sédimentation de l'expérience.

La notion de répétition recèle toujours une ambiguïté : celle-ci a été bien décrite par Guillaume (1947) dans ses recherches sur la formation des habitudes et l'on ne saurait mieux faire que reproduire la belle analyse qu'il lui a consacrée : « Mais il importe dès maintenant d'insister sur le carac- 
tère équivoque de cette notion (la répétition). Il y a contradiction entre l'idée de répétition, au sens rigoureux de répétition du même acte, et l'idée d'acquisition d'une façon d'agir nouvelle. Si on répétait toujours le même acte, il n'y aurait pas de changement; on n'apprendrait jamais rien. C'est parce qu'on ne se borne pas à reproduire qu'on apprend, qu'on progresse, qu'on s'adapte. Les gestes efficaces de la fin de l'apprentissage, avec leur économie d'effort et de mouvements inutiles, ne répètent pas les mouvements gauches et maladroits du début »(Guillaume, 1947, pp. 18-19). Cela ne concerne pas que le geste «moteur », comme le rappellent les études dans le domaine de la médecine sur les « effets classiques d'accélération [de l'action cognitive] par l'expérience [et] la fréquence d'exposition » (Ericsson, 2005, p. 235).

La répétition est particulièrement importante pour l'acquisition de l'expérience à des tâches où la composante sensorimotrice joue un rôle essentiel. C'est le cas notamment lorsqu'elle est associée à des exigences temporelles élevées : les sports, la conduite d'engins mécaniques, etc., ou à des exigences particulières de dextérité (couture de luxe, ébénisterie, etc.). Le rôle de la répétition dans ce type de tâches a été analysé dans les recherches conduites sur l'acquisition des automatismes (Leplat, 2008b, chap. 2), en particulier dans le domaine de la motricité. Le courant de recherche de l'énaction a également mis en évidence ce rôle essentiel du corps. Retenons ce raccourci qui donne bien l'esprit de ces recherches : «c'est le corps, comme on l'a dit souvent, qui "attrape" (kapiert) et qui "comprend" le mouvement » (Merleau-Ponty, 1945, p. 167). «L'habitude est un savoir qui est dans les mains et qui ne se livre qu'à l'effort corporel et ne peut se traduire par sa désignation objective » (id., p. 168). On pourrait renvoyer à la formule de Piaget et Inhelder (1966/2004) : « la mémoire d'un schème est ce schème » (p. 79). On retrouve aussi l'idée de l'automatisme comme compétence incorporée qui s'exprime dans l'action.

De nombreuses recherches ont été conduites sur l'effet de la répétition sur le temps d'exécution de tâches simples. Newell et Rosenbloom (1981) avaient recensé quelques-unes de ces recherches portant sur des tâches à dominante sensori-motrice aussi bien que cognitive. Il apparaissait que, pour toutes, les résultats s'ajustaient à un modèle dit « loi linéaire log-log de la pratique » qui exprimait la relation linéaire entre le log du nombre d'essais et le log du temps d'exécution, ce qui suggérait le caractère très général de la pratique. Les auteurs restaient néanmoins prudents sur la nature des mécanismes impliqués.

Ce qui est remarquable, c'est que les temps d'exécution des activités continuent à s'améliorer (c'està-dire à diminuer) après un nombre considérable d'essais. Dans une tâche élémentaire de détection, Shiffrin et Schneider (1977) constataient encore des modifications des temps de réponse après plus de 1000 essais. Norman (1993) notait que pour des tâches comme des calculs mentaux, la dactylographie, le jeu des instruments musicaux, des tâches sportives, « entre les stades initiaux de la performance des novices et la performance habile et polie (smooth) de l'expert, il y a des heures et des heures de pratique » (p. 29). Hayes (1985) avait apporté sur ce point une contribution intéressante par la recherche qu'il avait faite sur les productions d'artistes de haut niveau, en particulier des musiciens. Il concluait de ces recherches que « les résultats montrent d'une manière absolument claire que personne ne compose une musique remarquable avant une période d'environ dix ans de préparation musicale intensive » (p. 397) et il ajoutait que ces résultats signifiaient que même une personne dotée d'un génie tel que celui de Mozart ou de Beethoven « aurait encore besoin de dix ans ou plus d'intense préparation pour réaliser son potentiel » (p. 398). Il faut relever que des travaux relativement récents dans ce domaine font état de modifications cérébrales sur la longue durée de cette expérience (qui commence généralement très jeune, ce qui laisse une interrogation : plasticité initiale du cerveau ou effet de l'expérience sédimentée en tant que telle?).

Une question importante concerne les mécanismes sous-jacents à cette élaboration de l'expérience : à quelles conditions la répétition peut-elle conduire à l'acquisition? La psychologie de l'apprentissage permet d'apporter des éléments de réponse à cette question. On rappellera tout d'abord cette déclaration de Bartlett (1932) : « ce n'est pas la pratique qui apprend, mais la pratique dont les résultats sont connus. » Elle constitue aussi ce qu'on a nommé «principe de la connaissance des résultats ». Ce 
dernier a fait l'objet de nombreuses recherches dont on trouvera des inventaires et des classifications dans les manuels de psychologie de l'apprentissage classiques ainsi que dans Leplat (1970/2002) et George (1983).

La notion de résultat mérite aussi d'être analysée. Le résultat n'est utile pour l'acquisition que s'il peut être interprété, c'est-à-dire traduisible en termes d'action corrective ou préventive. C'est ainsi que la note donnée à un exercice peut être conçue comme traduisant l'écart entre le résultat obtenu et le résultat souhaité. Elle ne servira à l'amélioration de l'action future que si l'apprenant peut interpréter cet écart par rapport à son action, c'est-à-dire s'il peut rapporter cet écart à une caractéristique de l'action qu'il a mise en œuvre. En fait, il y a une double exigence : le repérage des indicateurs de la situation qui permettent de mettre en rapport le résultat et l'attendu, et l'identification d'une caractéristique de l'action propre du sujet.

L'étude du développement de l'expérience peut être abordée avec le modèle d'action proposé par Leontiev (1972) et souvent exploité dans les analyses du travail en ergonomie (Leplat, 2000). On n'en retiendra ici que le mécanisme du passage de l'action aux opérations. Une action est définie par son but conscient. La réalisation d'une action nouvelle se fera d'abord par l'élaboration et la réalisation d'actions élémentaires définies chacune par leur sous-but. Progressivement, avec l'expérience, ces actions élémentaires vont prendre le statut d'opérations, c'est-à-dire de moyens de réalisation de l'action principale. Quand on apprend à se servir d'un dispositif, on lit la notice en suivant la procédure qui définit les actions élémentaires; mais, progressivement, celles-ci s'agrègent et finalement le réglage final se fera par l'exécution d'opérations ordonnées au but général, sans qu'il ne soit plus nécessaire de fixer consciemment des buts intermédiaires. En s'intégrant à l'action finale, les actions élémentaires perdent leur statut et aussi leurs caractères propres. Il s'agit là d'une spécificité des expériences sédimentées.

\section{2.- Variabilité des conditions externes et variation de l'activité de l'opérateur}

La tâche à traiter ne se présente jamais exactement de la même manière ni dans un environnement parfaitement identique, et l'activité de l'opérateur n'est elle-même pas invariable. Pour désigner ces écarts lorsque des procédures explicites existent, les analystes parlent de variations (Leplat, 1987) ou de déviations (Kjellen, 1987). Une question essentielle est de déterminer à quelle référence rapporter ces écarts. Il existe, en effet, plusieurs références possibles : la norme fixée par la prescription, la référence fixée par un groupe d'opérateurs, etc. (Cellier, 1990). Ces normes ou références comportent en général des tolérances, c'est-à-dire que certains écarts sont considérés comme acceptables. Les erreurs désignent les écarts hors normes ou hors tolérances. Le sujet apprend ces normes et limites d'acceptation soit par des instructions (qu'il décide ou non d'observer), soit par l'expérience. Dans ce dernier cas, un mécanisme de base de cette acquisition est celui dit des essais et erreurs, bien connu depuis longtemps. C'est à travers la sanction des exécutions inacceptables que le sujet s'ajuste à la variabilité des situations et adapte progressivement sa procédure qui peut ainsi devenir satisfaisante.

Dans les tâches complexes, le sujet est amené souvent à définir lui-même un espace de travail acceptable ou sûr, soit que celui-ci ne soit pas préalablement défini ou définissable, soit qu'il veuille tester la qualité de celui qui est préalablement défini. Il le fera souvent grâce aux incidents, ceux-ci signalant qu'il est entré dans une zone critique. C'est par des essais répétés, plus ou moins systématiquement définis, qu'il cernera un espace de travail sûr. Rasmussen et al. (Rasmussen, Pejtersen, \& Goodstein, 1994) ont bien décrit un tel mécanisme. En formation, il sera donc utile d'introduire des variations dans les conditions d'exécution de la tâche pour amener le sujet à se construire un espace de travail pertinent bien balisé.

Deux grands types de variations peuvent être distingués qui ont été bien formalisés dans le cas de la régulation avec les deux modalités de cette dernière, la régulation fonctionnelle et la régulation structurale (Leplat, 2008b, chap. 1). Le premier concerne des variantes de la tâche qui peuvent être 
traitées avec le modèle de la tâche élaboré par le sujet pour les cas « ordinaires ». Un second concerne des situations pour lesquelles le modèle précédent n'est plus valable. Il en est ainsi des situations résultant de perturbations imprévisibles altérant les conditions habituelles du travail. Par exemple, un guichetier face à un client agressif qui veut lui faire résoudre un problème qui ne relève pas de ses attributions officielles. De tels cas exigent des solutions originales à élaborer dans des conditions difficiles, et l'adaptation n'est pas toujours réussie. Les ergonomes ont bien identifié ce type de cas. Ainsi Woods (2006) mentionne des cas manifestant une «sorte de défaillance de sous-adaptation où les gens persistent à appliquer les plans et les activités des manuels face à l'évidence d'un changement de circonstances qui exigerait une modification qualitative de l'évaluation, des priorités ou de la stratégie de réponse » (p. 22).

L'étude de la variabilité joue un rôle important dans l'analyse écologique du travail proposée par Norros (2004). Dans une recherche concernant la mise en œuvre d'une nouvelle technologie, Norros montre (chap. 4) l'existence de cette variabilité révélée par les perturbations (« disturbances ») qui interviennent dans l'activité des opérateurs qui ne peuvent plus être guidés par les instructions qui leur sont fournies pour les cas prévus. Ces perturbations traduisent la fragilité du système technique et les insuffisances de la conception. Les solutions que les opérateurs apportent face à ces perturbations à partir de leur expérience expriment la manière dont ils conçoivent la tâche qu'ils ont à exécuter. L'étude de ces solutions peut être exploitée pour améliorer la formation des opérateurs et la conception du système (p. 91 sq.). Norros donne de nombreux exemples de la place que peut tenir l'analyse de l'expérience dans l'amélioration continue de la conception d'un système technique. L'analyse que peut faire l'opérateur - seul ou assisté - de ces perturbations constitue un élément important pour l'amélioration de sa compétence : les analyses de Norros (2004) rejoignent sur ce point celle de Pastré (2005) avec son principe qu' « on apprend davantage en analysant son action qu'en la reproduisant » (p. 35).

En résumé, il faut noter que la variabilité des conditions externes de l'activité et celle de l'opérateur se co-déterminent. En même temps qu'il acquiert de l'expérience, celui-ci transforme les conditions externes qu'il prend en compte (son modèle des conditions externes), mais cette transformation suscite des mécanismes d'ajustement nouveaux. Norman (1993) a bien décrit un de ces mécanismes (tuning) : «Qu'est-ce que fait la pratique? Elle ajuste l'habileté, mettant en forme de mille petites manières les structures de connaissances de telle sorte que l'habileté qui dans des stades précoces exigeait une pensée consciente, réflexive peut maintenant procéder automatiquement, sur le mode subconscient, expérientiel. La pensée expérientielle est une pensée ajustée (experiential thought is tuned thought $) »(\mathrm{p} .29)$.

\section{3.- Les contextes non explicités de la tâche}

Il est devenu banal de dire que toute activité est située. Elle est située dans un contexte multiforme dont les rapports avec le noyau de la tâche sont plus ou moins directs. Ce contexte (physique, technique, organisationnel, social, etc.) contribue à façonner l'activité et à lui donner ses significations. Bruner $(1991,1996)$ a bien souligné ce phénomène à partir de la notion de culture. Pour lui, toute activité est culturellement située : « il est en effet impossible de comprendre l'activité mentale si l'on ne prend pas en compte l'environnement culturel et les ressources qu'il propose, ces mille détails qui donnent à l'esprit sa forme et sa portée » (1996, p. 7). Ce que Bruner dit de la culture vaut aussi pour l'expérience, à savoir qu' « elle est un produit de l'histoire » (1991, p. 27). Dans les situations de travail qui nous intéressent directement ici, les activités sont plongées dans un environnement sociotechnique qui en conditionne l'exécution. Citons notamment des traits de l'organisation : mode de contrôle, degré d'autonomie, climat de l'entreprise, rapports avec les autres opérateurs, avec les responsables, etc. L'expérience sédimentée est faite aussi de l'intériorisation de ces caractéristiques. Par exemple, l'expérience d'une secrétaire dépend non seulement de sa qualification par rapport à la tâche prescrite, mais aussi de ses rapports avec son directeur et avec ses collègues, des usages de 
l'équipement technique, etc. Il y a dans toute collectivité, dans toute entreprise des « coutumes », des façons d'agir dans différentes situations qui font reconnaître très vite un ancien d'un nouveau. Ces façons d'agir (expressions langagières, modalité de requête d'une autorisation) ne sont généralement pas explicitées ou explicitables et s'acquièrent par la pratique, souvent par une sorte d'imprégnation. Elles font partie de l'expérience et sont propres à un certain milieu. On retrouve ici les analyses de Fischer sur les contenus contextuels de la connaissance du processus de travail.

Clot propose de désigner ces caractéristiques de l'expérience sous le nom de genre qu'il a emprunté à Bakhtine : il a présenté et discuté ce concept dans plusieurs de ses publications (nous renvoyons en particulier à Clot, 1999; 2008). On reconnaît bien des traits de l'expérience dans certaines des définitions et commentaires qu'il donne de ce concept et de sa genèse. Le genre est « une sédimentation et un prolongement des activités conjointes antérieures et constitue un précédent pour l'activité en cours : ce qui a été fait auparavant par les générations d'un milieu donné, des manières dont les choix ont été tranchés jusque-là dans ce milieu, les vérifications auxquelles il a été procédé, les coutumes que cet ensemble rassemble» (Clot, 1999, p. 37).

L'expérience est finalement le produit conjoint des acquisitions issues de la tâche explicitée (celle à laquelle répond le sujet à partir de la tâche prescrite) et de celles issues des contextes dans lesquels celle-ci est insérée. La distinction de ces deux types de sources ne doit pas conduire à les opposer, car elles sont étroitement imbriquées. Le terme de « sédimentées » pour la modalité que nous venons de présenter renvoie à la transformation qui a lieu du fait de l'interaction avec une multiplicité de situations. Cette sédimentation procède de processus d'abstraction et de conceptualisation, de constitution de schèmes, d'automatisation de l'action, d'incorporation (Leplat, 1995). Si chaque situation traitée est singulière au moment de l'action, elle ne se conserve pas toujours individuellement dans la constitution de l'expérience professionnelle. C'est la modalité des « expériences épisodiques » que nous présentons dans la partie suivante qui renvoie aux situations qui ont conservé pour l'essentiel leur singularité.

\section{3.- Les expériences épisodiques}

C'est à la composante de l'expérience comme sédimentation que renvoient souvent les auteurs qui traitent du rôle de l'expérience dans la construction de l'expertise ou de la compétence professionnelle. Ainsi, dans un numéro spécial sur les avancées des recherches de psychologie cognitive sur l'expertise, Ericsson (2005) rappelle la nécessité, pour atteindre l'expertise dans le jeu d'échecs, d'un « intense engagement (...) et d'autres activités dans le domaine », sur la longue durée (au moins 10 ans) (p. 234). Il rappelle aussi sur les études en médecine les effets classiques d'accélération par l'expérience et la fréquence d'exposition. Dans ce numéro spécial sur l'expertise, il n'y a pratiquement qu'un article qui insiste sur les cas singuliers rencontrés dans l'expérience : Van Gog et al. rappellent que les modèles mentaux des experts dans la détection de pannes (trouble-shooting) contiennent un large corps de connaissances de défaillances précédemment rencontrées qui ont causé des (dys)fonctionnements similaires, ce qui leur permet de diagnostiquer et d'agir sur la base de cette similarité (Van Gog, Paas, \& Van Merriëndoer, 2005).

Nous allons développer ici cette dimension de la singularité des situations dans l'expérience, avec une analyse de ce que produit la pratique professionnelle en termes d'expériences épisodiques.

\section{Remarque : positionnement par rapport à des approches phénoménologiques du singulier}

Le vécu singulier est également au cœur des méthodes développées respectivement par Vermersch et par Theureau dans des cadres théoriques phénoménologiques. Vermersch présente l'entretien d'explicitation comme une technique pour « mettre en place des conditions d'accès à ce vécu [singulier] sur le mode du revécu »(Vermersch, 2000, p. 249 sq.). La méthode, dans laquelle les productions verbales du sujet sont les traces de l'action singulière, est présentée par exemple dans Vermersch 
(1990) et des exemples d'utilisation dans Vermersch et Maurel (1997). Les conditions temporelles d'accès au vécu singulier, et les propriétés qui permettent d'identifier une occurrence singulière de l'action ne sont pas étudiées. Notre position sur les expériences épisodiques est que si toutes les situations d'action sont singulières, elles n'en sont pas pour autant mémorisées à l'identique dans la constitution de l'expérience : certaines seulement y gardent leur caractère d'épisodes singuliers.

La méthode du «cours d'action » élaborée par Theureau se situe également dans une perspective phénoménologique. La visée n'en est pas moins de « dépasser le plan factuel de la description des occurrences pour atteindre [...] une typicalisation de l'activité du professionnel » (Durand, MeuwlyBonte, \& Roublot, 2008, p. 80). Theureau (2006) rappelle les « acquis de la méthode élémentaire » [cours d'action] qui sont «(1) l'activité humaine comme dynamique de couplage structurel et (2) les conditions d'une description symbolique admissible de la dynamique du couplage structurel » (p. 38). La «méthode développée » ajoute à l'objet théorique «cours d'action » d'autres objets théoriques, avec une extension à des " périodes d'activité discontinues s'étendant sur des empans temporels plus larges », qui " introduit, à travers la discontinuité de l'expérience, une hypothèse empirique supplémentaire de cohérence relative d'épisodes discontinus relatifs à une pratique à travers le temps » (pp. 48-52). Ces objets théoriques renvoient bien à un rapport entre continuité de la pratique et discontinuité des épisodes, mais dans un cadre spécifique qui rend discutable de faire un parallèle avec la relation entre « continuité » du vécu dans la modalité « sédimentée » de l'expérience et « singularité » dans la modalité « épisodique » que nous situons dans un cadre très différent.

Nous reprendrons pour la notion d'expérience épisodique une définition proposée dans Leplat (2008a) à propos de la notion de cas : " un objet, un événement, une situation constituant une unité d'analyse », cette unité étant « inscrite dans un contexte » (p. 182). C'est aussi « un objet clinique », " décrit autant que possible comme une totalité », dans une «étude intensive » (p. 183). La dimension temporelle y est prise en compte : « le cas peut revêtir une épaisseur temporelle plus ou moins grande ». Par exemple quand il s'inscrit dans un développement, il peut « devenir une histoire » (p. 183). Les expériences épisodiques sont d'abord le produit de telles unités vécues dans la pratique professionnelle.

Il peut toutefois s'agir aussi, comme nous le relèverons plus loin, d'épisodes vécus « au second degré » à travers leur récit par autrui (les « histoires », les « anecdotes » rapportées dans le travail), alors que les expériences sédimentées sont à la première personne.

Nous relèverons d'abord leur diversité de statut, de l'exception au paradigme. Nous discuterons de ce que peut apporter l'expérience d'un ou de cas singuliers à la compétence professionnelle, en relevant les limites temporelles impliquées dans les processus de mémorisation. Le tissage social des expériences épisodiques sera également considéré à partir de la fonction du récit d'épisodes (anecdotes) ou de la collection organisée de cas, fournissant les bases du retour d'expérience (REX) ou de la résolution de problèmes basée sur des cas (case-based reasoning: CBR).

\section{1.- L'épisode : de l'exception au paradigme}

Les expériences épisodiques sont donc issues de situations singulières, considérées à la fois dans leur globalité et dans leur particularité. Certes, toute situation est singulière, mais dans les expériences épisodiques la mémoire du vécu intervient en tant que telle, dans un contexte unique. En fait, il existe différentes acceptions du terme « épisode » auquel renvoie la modalité de l'expérience que nous considérons ici. Il existe différents points de vue, souvent implicites, sur ce qui constitue un épisode.

L'acception la plus fréquente est celle d'incident, où l'accent est mis non par tant sur la singularité de la situation que sur un caractère de perturbation voire de rupture de la régularité des événements ou de la routine des actions. La dimension de «problème » est sous-jacente à la notion de «cas » dans toute la littérature sur le raisonnement par cas (case-based reasoning), qui interroge en particulier le 
passage de la connaissance de cas singuliers au traitement de situations nouvelles. La focalisation sur cette dimension problématique est explicite dans les discussions sur la formation par la résolution de problème (problem-based training), en particulier la productivité de cette approche a été discutée en médecine (toutefois les problèmes auxquels il est fait référence sont davantage des types de problèmes ou des scénarios types que des épisodes vécus ou rapportés).

« Incidents » et « problèmes » n'occupent d'ailleurs pas des positions interchangeables. L'incident renvoie fondamentalement à une perturbation de la performance du point de vue de la sécurité (ou plus largement de la fiabilité). Le problème renvoie davantage à l'exigence de la situation en termes de compétence de l'acteur qui y est confronté. On parle d'ailleurs de résolution de problème.

Il faut cependant relever que la notion d'incident critique introduite par Flanagan (1954) reprend des éléments qui renvoient autant au contexte spécifique d'une situation particulière qu'à l'exigence d'un tel incident du point de vue des compétences. L'un des buts de Flanagan était d'aboutir après analyse d'un ensemble d'incidents critiques rapportés à l'identification des « exigences critiques » pour les acteurs du domaine considéré (officiers militaires, pilotes de ligne, personnels de recherche, contrôleurs aériens, opérateurs de l'industrie manufacturière...) et établir des procédures de sélection. La formation n'y était pas évoquée. Peu après, une étude de Gendre (1968/1990) fondée sur la technique des incidents critiques montrera des évolutions de la nature des incidents cités avec l'ancienneté professionnelle, mais pas au cours de la formation initiale.

Il faut relever que la technique de l'incident critique, qui utilise des «protocoles anecdotiques » (p. 167), n'est pas centrée sur le négatif (l'incident mettant en cause la sécurité, par exemple, ou le problème montrant la limite de compétence de l'opérateur). Cette technique appelle en effet systématiquement les participants aux études à expliciter des incidents spécifiques de comportement efficace ou inefficace dans un contexte donné, en se centrant sur des épisodes précis. Elle fait l'objet d'un chapitre de l'ouvrage de Bisseret et al. sur « les techniques pratiques pour l'étude des activités expertes » (Bisseret, Sebillotte, \& Falzon, 1999, pp. 124-132). Les auteurs expliquent l'application de la méthode illustrée par la présentation d'incidents critiques recueillis dans des études françaises d'ergonomie.

La technique des incidents critiques a été largement reprise depuis Flanagan (dont le texte princeps, dans le Psychological Bulletin de 1954, est d'ailleurs désormais en ligne sur le Web, avec une base de données produite par l'APA de plus de 300 pages). Elle a été transposée en une « méthode de la décision critique » (Critical décision method, $C D M$ ) pour faire expliciter la connaissance experte dans des situations complexes (essentiellement des situations dynamiques) (Hoffman, Crandall \& Shadbolt, 1998). Il s'agit d'une méthode de questionnement d'experts visant à stimuler le rappel de cas saillants, qui ont impliqué des décisions critiques, exigeant de l'acteur la pleine application de sa connaissance experte. Les auteurs soulignent que la recherche sur les décisions critiques « a une base théorique forte, ce qui est une raison pour pouvoir l'utiliser comme étude de cas » (notre traduction, p. 256).

Une revue critique de l'utilisation de la technique de l'incident critique dans le domaine des services (Gremier, 2004) met en évidence une hétérogénéité dans la méthodologie des travaux qui s'y sont référés (141 articles analysés). Nous garderons plus loin la référence à l'article «fondateur » de Flanagan pour des comparaisons « à méthodologie invariante ».

Mais les expériences épisodiques ne se limitent pas pour un acteur aux ruptures de la routine, à l'exceptionnel ou aux situations problématiques. Elles peuvent aussi concerner des situations particulières typiques, des exemples. Un épisode vécu ou rapporté peut alors être illustratif de toute une classe de cas et jouer un rôle de paradigme. Lorsqu'il s'agit d'un objet " clinique », mémorisable comme une totalité avec son contexte, visant l'exhaustivité, le cas devient une ressource d'interrogation possible du réel de l'action, au-delà de la description nécessairement schématique du type de cas dont il est le paradigme. On parle d'ailleurs souvent de « cas d'école », ce qui insiste sur son potentiel de formation. D'autres épisodes produisent sur l'acteur des effets d'expérience pour avoir 
été la première rencontre avec un type de situations, pour être une «première fois que... $»^{3}$.

\section{2.- L'apport de l'épisode « cas singulier » à la compétence professionnelle}

La question de la généralisation est au cœur de la discussion sur la place des expériences épisodiques, singulières, dans le développement des compétences : qu'apprend-on d'une situation particulière vécue qui dépasse sa singularité ? Dans quelle mesure étendre les conclusions?

Au-delà de la problématique de la résolution de problème à partir de cas (case-based reasoning) dont le développement concerne de manière majeure la gestion collective de la connaissance, via des systèmes informatisés (voir infra), le rôle des « cas » a été spécifiquement discuté dans certains domaines de la pratique professionnelle. Il s'agit plus particulièrement de situations où le but de l'activité est de prendre des décisions sur des personnes, au « cas par cas », selon une expression bien parlante (on peut penser au droit, avec la notion de « jurisprudence », mais aussi aux processus d'embauche, à ceux de la clinique, etc.). Un autre domaine est celui de l'activité de chercheur ou de praticien dans le champ des sciences sociales (Passeron \& Revel, 2005). Dans les domaines où il s'agit de prendre des décisions sur des personnes, la problématique des cas s'exprime comme «casuistique » (voir par ex. Jonsen \& Toulmin, 2005), sa visée est d'abord - voire seulement - pragmatique. Dans les domaines des sciences sociales, le questionnement concerne la productivité d'une méthode d'étude basée sur des cas singuliers. Il peut s'agir de produire des savoirs de nature scientifique dans le cas de la focalisation épistémique d'une recherche. Il peut aussi s'agir de développer les compétences du chercheur ou du praticien, avec une focalisation pragmatique (Leplat, 2008a; Clot \& Leplat, 2005).

Un cas renvoie à des principes ou des processus généraux connus ou recherchés : il en est une particularisation. Il est associé à une notion de contexte qui définit ses conditions particulières. Il fait intervenir des variables qui peuvent être bien plus nombreuses, mais aussi moins bien définies, que les variables explicitées dans des principes généraux (théorie ou modèle). Un cas est analysable « en profondeur », selon plusieurs points de vue, méthodes, ou sources d' "évidence ». L'unité d'analyse qu'est le cas « déborde » alors la formalisation en termes de modèles ou règles, qui schématisent toujours le réel (en quoi d'ailleurs elles sont des représentations opérationnelles).

À partir du traitement d'un cas en vue de sa résolution, son exploitation pour « en apprendre plus » pose le problème de la généralisabilité du cas, qui est généralement de type «naturalistique » (pour reprendre un terme issu de l'approche de Klein sur la prise de décision : «naturalistic decision making » - Klein, 1989) et non déductive. Cependant, un ensemble de cas (un "espace de cas » (Goldenberg \& Mason, 2008) peut avoir des propriétés qui permettent une exploitation déductive. Gomm, Hammerseley et Foster (2000) ont souligné cette possibilité : il en est ainsi lorsqu'on veut, par exemple, montrer l'hétérogénéité d'une population, et repérer des dimensions pertinentes de cette hétérogénéité ( «significant likely dimensions of heterogeneity of a population »). Il en est de même pour montrer l'existence de contre-exemple(s) ou d'exception(s) à une règle générale (ce peut être «l'exception qui confirme la règle »... ), ou pour produire une preuve («evidence ») à propos de la typicalité d'un cas ( «in support of claims that the case(s) studied are typical (or atypical) in relevant aspects ») (op. cit, p. 111). A contrario, l'épisode comme paradigme d'une situation peut devenir l'instrument de contrôle d'une règle ou de récupération d'une procédure dont l'existence est connue.

Toutefois, dans un domaine comme les mathématiques, un cas ou, comme on le définit le plus souvent, un « exemple » peut être traité d'entrée de jeu comme générique. Conne (2008) attire alors notre attention sur les différences de traitement selon qu'il s'agit d'un mathématicien qui connaît déjà le domaine, et va pouvoir traiter déductivement la valeur générale de l'exemple, en relevant que les opérations accomplies ne dépendent pas de la particularité de l'exemple, ou d'un débutant qui ne peut s'appuyer que sur une abduction, en posant une hypothèse à partir du cas.

3. La littérature sur l'apprentissage animal a montré la possibilité d'existence d' «apprentissage en un coup » dans le cas de situation aversive. L'écologie a par ailleurs mis en évidence le phénomène d'imprinting lié à l'objet d'une première rencontre (cf. la célèbre expérience de Conrad Lorentz avec les oies au sortir de l'oeuf). 
L'épisode, comme singularité, peut enrichir la représentation du monde de l'action. Il peut tout d'abord, et surtout pour le débutant, «donner chair au modèle ». On peut penser ici à une relation entre épisode et modèles d'une nature un peu analogue à celle que Vygotsky établit entre concept quotidien chargé de sens et concept scientifique (Vygotsky, 1997, pp. 271-413). Il faut ajouter que les expériences épisodiques ne présentent pas seulement un apport pour la résolution du problème (problem solving), mais aussi pour la définition de (nouveau) problème (problem setting), par exemple dans l'analyse réflexive d'un épisode où le « connu » a été mis en échec.

On peut aussi chercher, pour étendre un modèle ou une théorie, une "généralisation analytique » d'un cas au sens suivant : à partir de l'événement particulier qu'est le cas, on veut identifier la mise en œuvre de principes théoriques généraux. Le cas peut aussi servir de «banc d'essai » pour tester une proposition, trier parmi des hypothèses plausibles, des règles, des principes, ou les raffiner. Il faut relever aussi la place du cas dans la dimension « argumentative » de l'activité, non seulement pour réfuter, mais aussi pour emporter l'adhésion sur une conclusion. Il en est souvent ainsi pour l'expression «par exemple».

La relation entre le cas singulier et la connaissance générale est aussi posée dans le sens inverse. Ainsi, Granger (1967) souligne la relation dialectique entre savoir (général) et pratique dont l'articulation est le lieu de la connaissance clinique : «la science ne peut viser valablement l'individu qu'après un très long détour ». Widlöcher (1999) exprime la même idée pour le domaine de la psychologie clinique : «le clinicien forme son savoir sur un cas individuel par accumulation de données et références à des types ».

En fait, il y a deux finalités dans l'étude d'un cas, l'une consiste à trouver une solution à un problème particulier, l'autre à trouver le principe d'élaboration d'une solution. Il y a ainsi une dialectique du travail sur les expériences singulières entre une étude « nomographique », visant des lois générales, des invariants, et une étude «idiographique », c'est-à-dire visant la singularité : dans une étude de cas, on peut voir "la considération d'un individu en lui-même, c'est-à-dire idiographiquement, et sa considération comme porteur d'un type, c'est-à-dire comme membre d'un domaine nomothétique » (Smith, Harré, \& Van Langenhove, 1995, p. 65, traduit par Leplat, 2008a, p. 186).

Les débats sur le rôle des cas ont été nombreux en sciences sociales. En ergonomie et en psychologie du travail, Clot et Leplat (2005) en ont analysé l'historique et le fonctionnement, montrant que la méthode des cas a pu (et peut) constituer « un moyen privilégié d'acquisition des connaissances sur les situations de travail et en particulier pour la conception des interventions » et qu'elle «s'est enrichie en devenant un instrument de transformation des situations de travail, sans perdre sa fonction d'acquisition de connaissances » (p. 290).

Il nous semble ainsi que le questionnement sur le rôle des cas dans l'activité scientifique peut être transposé à d'autres champs d'activité professionnelle, avec les précautions qui sont nécessaires pour passer d'une focalisation à dominante épistémique à la focalisation à dominante pragmatique qui est celle de la plupart des activités auxquelles s'intéresse la didactique professionnelle.

\section{3.- Apprendre des expériences épisodiques : de l’individu au tissage social de la continuité des savoirs}

Des auteurs ont abordé ce point par le questionnement du rôle des cas dans la production de la connaissance : ils relèvent en particulier que les cas fournissent un «ensemble de données empiriques ». Ce terme d' « empirique » renvoie à une problématique de production de connaissances générales, ou à celle de constitution d'une base pour la résolution de problèmes, dans le contexte des travaux sur le raisonnement à base de cas. La question a été particulièrement étudiée dans le domaine de la médecine, dans des cadres d'aides informatiques à la conception, l'unification et l'utilisation de bases de cas (cf. Bichindaritz, 1995 et Bichindaritz \& Marling, 2008). Plusieurs problématiques se croisent : l'utilisation par autrui des expériences épisodiques médiatisées par le discours en relation 
de face-à-face; le retour d'expérience au sein d'un système par l'organisation de rapports d'incidents $(\mathrm{REX})$; la résolution de problème à partir de cas déjà résolu $(\mathrm{CBR})$.

\section{- La transmission des expériences épisodiques}

Les expériences épisodiques «à la première personne » jouent potentiellement un rôle dans le développement professionnel individuel. Elles peuvent aussi être adressées à d'autres membres de la collectivité professionnelle et médiatisées par une mise en récit (storytelling). C'est à quoi renvoie le terme « anecdote ». Soulignons ici que pour qu'il y ait récit, il faut une remémoration délibérée de l'épisode, donc que celui-ci ait un caractère conscient (ou conscientisable). Il faut aussi « les mots pour le dire ».

La transmission d'expériences épisodiques par le récit dans un collectif peut alors conduire au développement d'autrui (novice ou bien acteur déjà compétent). Un des premiers à avoir étudié cet impact en situation professionnelle, Baerentsen (1996) a montré que ce qu'il appelle «episodic knowledge » est effectivement utilisé pour le traitement de nouveaux incidents, que l'épisode incidentel ait été directement rencontré par l'acteur ou qu'il ait été rapporté. En « amont » du traitement de situations nouvelles, les expériences épisodiques avec des cas schématisés en prototypes ou racontés dans leur singularité contribuent à la communication dans la conception (Erickson, 1995).

\section{- L'utilisation en formation des expériences épisodiques rapportées}

Les expériences épisodiques des « anciens » peuvent être utilisées délibérément pour la formation des «nouveaux ». Marchand a ainsi analysé l'utilisation des « récits expérientiels » - et la place des savoirs qui y sont exprimés - dans l'apprentissage de la gestion des risques, dans le cas de la formation de pilotes militaires (Marchand, 2009; Marchand \& Falzon, 2006). L'identification de «pièges » dans ces situations à risque est un but essentiel des anecdotes. Dans un tout autre domaine, la place des récits d'intervention dans les situations pédagogiques en formation à la pratique ergonomique est l'objet d'une étude en cours, en relation avec l'impact de ces récits sur les étudiants futurs ergonomes (Beaujouan \& Daniellou, 2009).

L'échange d'histoires peut être une stratégie de développement des compétences lorsque sont en jeu des processus qui ne peuvent se produire dans une formation hors travail. Dans une revue de didactique sur la formation des infirmières, un article analyse une telle stratégie de formation (Hunter \& Hunter, 2006). Les auteurs ont montré le rôle très important, voire irremplaçable, des histoires rapportées au sein d'un collectif les accueillant avec l'intention d'en tirer des leçons. L'explicitation des émotions et de la gestion qui a pu en être faite - qu'il s'agisse des émotions du soignant ou de celles du patient - permet une clarification émotionnelle. Cette dernière est un composant du développement professionnel lorsqu'il s'agit de domaines impliquant des relations interpersonnelles fortes (relations de soin, mais aussi relations de service, enseignement...). Des expériences épisodiques peuvent également être transposées en scénarios pour la formation (Plaza, 2009).

\section{- Expériences épisodiques et retour d'expérience (REX) dans les organisations}

La problématique de l'organisation du retour d'expérience a été développée pour les systèmes à risque, qui cherchent à organiser des rapports d'incidents vécus par les opérateurs pour en tirer des connaissances sur les risques permettant d'améliorer la fiabilité et la sécurité. Il s'agit ici de l'apport des expériences épisodiques cumulées du point de vue de l'organisation elle-même, et non plus du point de vue individuel, dans l'expérience personnelle ou celle médiatisée par le récit à autrui. Deux voies contrastées peuvent être développées : organiser la structure des rapports d'incidents selon les facteurs susceptibles d'avoir joué un rôle dans l'incident ou exploiter la richesse d'incidents singuliers en s'appuyant sur les connaissances d'experts du domaine. Dans une recherche sur l'activité des enquêteurs de la navigation aérienne en charge d'analyser des incidents, il a été observé que les enquêteurs expérimentés exploraient des possibles « autour » de l'incident effectif, par une activité inférentielle de raisonnement contre-factuel, enrichissant ainsi leur analyse (Barriquault \& Rogalski, 2001). Pour faciliter l'évocation de scénarios potentiels, une méthode de questionnement a été éla- 
borée (Méthode d'élaboration de scénarios potentiels : MESOP). MESOP articule les données du scénario effectif étudié et les écarts de variables de contexte, en termes de dégradation ou au contraire de renforcement de la sécurité. La productivité de l'analyse d'épisodes en est renforcée chez des enquêteurs débutants (Barriquault \& Rogalski, 2003 ; Barriquault, 2005).

Le choix de l'épisode, dont le récit, sera objet de travail pour la formation, l'intervention sur la manière dont il est rapporté, ainsi que l'analyse de la manière dont il est entendu et ensuite travaillé, sont affaire de décision didactique. Il est important d'analyser les transpositions qui ont nécessairement lieu du fait de la situation de la formation, et donc du fait que les acteurs en formation n'ont pas ou peu eu d'occasions de se représenter des contextes variés d'action, contextes qui conditionnent l'interprétation et la portée des expériences rapportées.

\section{- Les conditions de la productivité des expériences épisodiques partagées}

L'impact des expériences épisodiques rapportées sur le développement des compétences collectives ou des connaissances organisationnelles ne va pas de soi. Des conditions ont été relevées quant à la qualité des rapports d'incidents ou des « histoires » de métier rapportées. Par exemple, dans un contexte de développement d'un système utile de rapport d'incidents dans le domaine hospitalier, Rooksby et al. déclarent que « le rapport utile est celui qui ne présente pas simplement une erreur ou une faute (error or mistake), mais propose une histoire qui peut être discutée, interprétée, et travaillée » (Rooksby, Gerry, \& Smith, 2007).

Une autre condition de la productivité de l'analyse d'épisodes a été soulignée pour les systèmes à haut risque, dans lesquels les incidents sont si rares qu'il est utile d'utiliser ceux d'autres systèmes. Il faut alors élever le niveau d'analyse des incidents, en remontant aux causes dans leur principe et non dans leur réalisation particulière, pour qu'il soit possible d'en tirer des leçons plus générales, au-delà du seul domaine concerné par l'incident ou l'accident. Cette condition a été soulignée à propos de l'analyse visant la sécurité des procédés chimiques à risque élevé (Hendershot, 2008) : l'utilisation de la connaissance ainsi produite peut concerner aussi bien l'ingénieur de prévention que l'entreprise dans son ensemble. L'auteur souligne par ailleurs la nécessité de faciliter ce changement de niveau de description et d'analyse des cas.

\section{- Expériences épisodiques et raisonnement à base de cas (CBR) dans les collectifs et les organisa- tions}

Les épisodes de problèmes résolus, rassemblés dans des bases de cas, sont un outil pour une communauté de pratique ou une organisation, pour constituer une expérience collective sur la base de laquelle pourront être recherchées des solutions à de nouveaux cas.

L'intelligence artificielle a été au centre du développement des recherches sur le CBR (auquel on ne peut pas identifier le raisonnement humain par analogie - cf. 4.2.).

En conception, la programmation informatique a été l'un des domaines où cette question a fortement été posée (cf. Burkhardt \& Détienne, 1995). Plus généralement, Darses et al. (Darses, Détienne, \& Visser, 2004) ont souligné les besoins de systèmes (informatisés) d'assistance aux concepteurs pour utiliser ce qu'on peut considérer comme un collectif d'expériences épisodiques. Cela concerne la constitution de bases de cas, la manière de conserver la mémoire de la logique de conception, et l'apport de l'intelligence artificielle dans l'exploitation de telles bases. Combattre l'explosion combinatoire des comparaisons systématiques (que ne fait pas le sujet humain) entre cas nécessite par ailleurs une organisation adéquate, dès lors qu'il y a une certaine « densité » de l'espace des variables définissant les cas. Un exemple intéressant d'une méthode implémentée en IA pour la conception de processus chimiques peut être trouvé dans (Negny \& Le Lann, 2008). Faire face au vieillissement d'une base de cas est un autre problème, produit justement par l'absence même de l'oubli. Le problème de la maintenance des bases de cas a été soulevé en particulier pour la médecine par Bichindaritz et Marling (2008, pp. 131-132) en relation avec l'évolution des connaissances médicales et des techniques thérapeutiques. 


\section{- Élaboration de méthodes à partir d'expériences épisodiques}

L'élaboration de méthodes comme invariants des traitements (de situations-problèmes) qui ont « réussi » est une autre des médiations du passage de l'épisode personnellement expérimenté à une connaissance générique. La méthode, en ce sens, est une forme de modèle-référence pour l'organisation de l'activité, à l'instar de la procédure elle constitue un outil cognitif, à sa différence elle présente à l'acteur les actions à conduire, mais le laisse en charge du « comment » (des opérations). Une méthode est un outil cognitif « pour soi » lorsqu'elle est propre à celui qui l'élabore, et si elle est partageable et partagée, elle devient un outil cognitif collectif. Ainsi, le rôle des méthodes pour la compréhension mutuelle de l'action dans les collectifs de travail a été relevé par Hoc et Rogalski (1992). L'existence de méthodes enseignées et l'exigence de la maîtrise de ces méthodes pour le recrutement ont été particulièrement marquées dans le domaine de la conception de systèmes informatiques et celui de l'ordonnancement (la méthode MERISE et la méthode PERT en sont des « fleurons »). En fait, la question est posée dans les situations « ouvertes », où les solutions ne peuvent être procéduralisées, et où la «densité » des variables rend l'utilisation directe d'expériences épisodiques insuffisamment productive. Karsenty (2001) a ainsi proposé des méthodes pour la création de mémoires de projets en conception.

\section{4.- Limites, et condition d'efficacité des expériences épisodiques}

Nous avons souligné dans quelle mesure les expériences épisodiques sont des ressources tant individuelles que collectives. Il faut aussi relever leurs limites et conditions de productivité. Il en est ainsi des limites temporelles dans la remémoration des expériences épisodiques, de l'importance de la compétence de l'acteur, et des possibles impacts émotionnels négatifs.

\section{- Les limites temporelles dans la remémoration des expériences épisodiques}

Le développement professionnel à partir d'expériences épisodiques n'est pas nécessairement réalisé : il existe des «pathologies » de l'expérience pour l'acteur; il y a des conditions pour que l'expérience des anciens serve aux nouveaux, à travers une médiation didactique, au travail ou en formation.

Un élément évidemment central pour l'utilisation des expériences épisodiques est la mémoire qu'en gardent l'acteur ou l'auditeur au fil du temps. Dans son travail sur les incidents critiques, Flanagan rapporte des effets temporels de remémoration : plus d'incidents sont rapportés pour le passé proche; les incidents rapportés au bout d'un temps assez long sont "sélectionnés ». Il a montré que les acteurs ont tendance à inclure une proportion plus grande de situations dramatiques, dans le cas du contrôle aérien (Flanagan, 1954, p. 174) ou celles présentant une déviation appréciable par rapport à la normale, dans le cas d'une industrie manufacturière, que ce soit pour les comportements efficaces ou inefficaces (p. 179).

\section{- L'importance de la compétence dans la constitution des expériences épisodiques}

Flanagan rapporte aussi (p. 180 sq.) des effets de l'expertise de celui qui fait l'expérience d'un épisode particulier (comme acteur, observateur ou auditeur). Il interprète ces effets par le rôle de la compétence pour évaluer la contribution d'une action déterminée au but général de l'activité, et il insiste sur «l'importance d'identifier [ce] but général d'une activité pour recueillir des incidents significatifs et les analyser » (p. 269). Beaucoup plus récemment, O'Hare et al. (O'Hare, Mullin, Wiggins, \& Molesworth, 2008; O'Hare \& Wiggins, 2004) ont montré, dans le cas de l'aviation, que l'utilisation de cas passés n'est pas automatique. Elle dépend des compétences des acteurs, ce qu'ils interprètent avec l'hypothèse que «le problème clé pour les décideurs est d'identifier quels cas ou instances antérieurs sont les plus pertinents pour la situation en cours » (notre traduction). L'évaluation des indices adéquats pour caractériser un épisode particulier est liée à la compétence des pilotes : ces auteurs (Wiggins \& Hare, 2003) ont ainsi conçu et validé un logiciel pour la formation (de pilotes non professionnels) à l'évaluation d'indices annonçant des conditions météorologiques devant les conduire à modifier leur plan de vol. 
On peut rattacher cet effet de la compétence à des propriétés de la compréhension des situations mémorisées : dans la mesure où « comprendre [c'est] construire une représentation » (Richard, 2004, Chap.4, pp. 109-178), on doit s'attendre à l'impact des ressources en matière de catégorisation et de conceptualisation sur l'encodage des situations qui resteront mémorisées, et ultérieurement sur le processus de remémoration. Toutefois, des situations peuvent être mémorisées au niveau des traits de surface («verbatim memory ») - même pour des cas qui n'ont pas «fait sens » pour le sujet - et faire ultérieurement l'objet d'une nouvelle interprétation.

\section{- L'impact du caractère émotionnel des expériences épisodiques}

L'expérience ne se joue pas seulement sur le plan cognitif : elle existe aussi sur le plan émotionnel. Une expérience épisodique où le sujet lui-même a été mis à l'épreuve possède un potentiel d'impact dépendant de la manière dont le sujet a vécu cette situation, qu'il ait ou non « réussi son schibboleth $»^{4}$. L'expérience a certes un impact en elle-même, mais cet impact dépend de manière décisive de ce qui est élaboré chez et par le sujet à partir de cette expérience. Cela est au cœur de la problématique de la résilience en psychologie clinique ${ }^{5}$.

L'élaboration sur cette mise à l'épreuve est un facteur de l'impact de l'expérience, en termes de mémorisation (et donc d'utilisation délibérée de l'expérience) comme en termes de déterminant éventuel de l'action future. Drivdahl et al. ont montré comment pouvaient ainsi se créer de « fausses mémoires » (Drivdahl, Zaragoza \& Learned, 2009). La mémoire de l'épisode peut aussi être « oblitérée », y compris dans des cas où le sujet s'est bien sorti de la situation, mais a ressenti de la peur, de l'inconfort ou une mise en cause de son image.

Qu'il y ait ou non mémorisation (accès délibéré à la mémoire), l'expérience épisodique peut être le déterminant d'une action peu appropriée au contexte en cours. On peut en trouver un exemple (Rouve \& Ria, 2008) à propos d'un enseignant débutant, marqué par la première classe qu'il a prise en charge. L'année suivante, lors du « démarrage » d'une leçon de physique, cet enseignant, écrivant au tableau, est impressionné par l'enseignant de la salle voisine qui contrôle mal sa classe; il intime alors le silence à ses propres élèves bien que sa classe soit tout à fait calme. En fait, il a gardé l'empreinte de son expérience antérieure de contrôle difficile de ses élèves, qui l'inquiète quant à son avenir d'enseignant (à propos du collègue voisin, il commente « je n'ai vraiment pas envie de devenir comme ça ! »). L'enseignant a par ailleurs conscience du décalage avec la situation présente : «même si c'est calme, j'ai l'impression que ce n'est pas cadré, c'est dans ma tête ». Dans leur discussion générale, Rouve et Ria relèvent que les enseignants débutants (novices) tirent de leur expérience « des indices typiques de situations déjà vécues ». La typicité peut être l'objet d'un biais de représentativité qui peut être accentué s'agissant de situations qui ont eu une charge émotionnelle négative.

\section{4.- L’articulation entre expériences sédimentées et expériences épisodiques}

Les expériences sédimentées ont aussi bien des traits de compétence incorporée et des traits de connaissances sémantiques explicitables. L'expérience sédimentée a les traits d'une compétence incorporée dans la mesure où elle se révèle essentiellement dans l'action : comme elle, elle peut être

4. Schibboleth: épreuve décisive qui permet de juger de la capacité d'une personne. Il s'agit d'un mot hébreu, utilisé pour reconnaître des étrangers à la manière dont ils ne le prononcent pas correctement (dans un récit de l'Ancien Testament). L.J. Calvet rapporte une anecdote de cet ordre se passant au cours de la révolte à Palerme contre l'occupant français -en 1582- à propos du mot sicilien «ciciri » (La guerre des langues, Hachette, 1999, p. 41).

5. La sémantique du terme « résilience » a été étendue à des contextes dans le champ de l'ingénierie (Woods, 2006), où il s'agit plutôt de robustesse du point de vue de la fiabilité et de sécurité des systèmes socio-techniques. 
difficilement verbalisable, ou ne pas être consciente. Les traits de connaissances sont ceux qui sont explicitables dans des représentations symboliques, langagières ou non langagières (schémas, par exemple). Vergnaud parle respectivement de composantes procédurales et déclaratives de la connaissance (Vergnaud, 2007, p. 36). Deux problématiques de psychologie renvoient à la dimension « historique » de l'activité, et renvoient aussi à l'articulation entre expériences sédimentées et expériences épisodiques : il s'agit, d'une part, des études sur la relation entre mémoire sémantique et mémoire épisodique, et d'autre part, des relations entre types et occurrences dans les processus de catégorisation et de raisonnement par analogie.

\section{1.- Expériences et types de mémorisation}

Une composante des expériences sédimentées est de nature conceptuelle, impliquant des relations entre concepts et indicateurs (les indices ou les traits pertinents de types de situations). Il lui correspond une mémoire sémantique (ce que le sujet sait du monde). Les expériences épisodiques sont par nature contextuelles, et il leur correspond une mémoire de type épisodique. Cette distinction de types de mémoire est ancienne en psychologie.

Mémoire épisodique et mémoire sémantique sont deux formes de connaissance auxquelles on a accès par le langage. Si elles fonctionnent selon des modes distincts, elles sont néanmoins interdépendantes. Ainsi, l'accès à la mémoire sémantique peut faciliter la reconnaissance épisodique d'incidents, tandis que, à travers la répétition de situations, de nouvelles informations sont abstraites de leur contexte épisodique et représentées en mémoire sémantique.

Nous interprétons ce dernier point comme un effet de sédimentation d'expériences épisodiques. Ce processus pourrait bien être lié au passage du cas-épisode (celui de l'expérience épisodique) au cas comme type (celui du raisonnement à base de cas). On rejoint la question du rapport entre épisode comme complètement contextualisé (occurrence) et analyse sémantique de l'épisode (le type). On peut peut-être rattacher à ce type de processus le phénomène d'amalgame entre épisodes (blended episodes), décrit en particulier par des pilotes comme le fait que des épisodes « voisins » peuvent donner lieu à un souvenir de type épisodique, mais qui mêle des éléments contextuels appartenant à des épisodes différents (ce ne sont pas tout à fait des types, ce ne sont plus tout à fait des occurrences). Par exemple, un épisode « inducteur » évoquera pour l'un d'eux « toutes (m)es collisions volatiles » (Marchand, 2009, p. 160).

La notion de «mémoire d'essence » (gist memory) a été élaborée pour rendre compte d'un processus de mémorisation entre occurrence et type, dans une modélisation de la catégorisation avec des ensembles flous (Brainerd \& Reyna, 1990). Elle s'oppose à la mémorisation «textuelle » des détails de l'occurrence (verbatim memory). Selon la théorie initiée par Brainerd et Reyna, deux traces indépendantes, "d'essence" et "verbatim", sont encodées pour tout événement mémorisé. La mémoire "d'essence" est une représentation floue de la signification et de la structure sous-jacente à l'événement. La trace de la mémoire "verbatim" est une représentation exacte au niveau des traits de surface de l'événement.

Du point de vue de l'économie de la mémorisation, Goldsmith et al. (Goldsmith, Koriat, \& Pansky, 2005) se sont demandés si l'oubli différentiel de l'information selon les deux types de traces peut être exploité pour la régulation de la qualité du rapport de l'information au cours du temps. Leurs résultats suggèrent que lorsque des acteurs ont la possibilité de contrôler la « granularité » de l'information lors de leur remémoration, ils ont tendance, pour maintenir un niveau stable de qualité de cette remémoration, à donner des réponses « à plus gros grain » sur du plus long terme. Les notions d'image opérative (Ochanine, 1978) et de mémoire opérationnelle (Bisseret, 1995, pp. 124-125) renvoient à des processus de sélection et de focalisation qui vont dans le même sens de conservation de l'essence d'un objet ou d'une situation du point de vue de l'action à conduire. On peut penser qu'un tel mouvement au sein des expériences «épisodiques » permet de renforcer la productivité des épisodes pour l'action ultérieure, sans en perdre la « chair». 


\section{2.- Occurrences, types, analogie}

Les deux modalités de l'expérience fonctionnent dans les processus de catégorisation; les principes abstraits des expériences sédimentées peuvent converger, mais aussi diverger quand il s'agit de décider sur un cas singulier; la dimension sémantique liée aux expériences sédimentées, et les cas singuliers retenus dans les expériences épisodiques ont une place dans les traitements par analogie de situations nouvelles. Ce sont ces mises en relation que nous présentons ci-dessous.

\section{- occurrences, types, et mémoire de "l'essence » d'un événement}

Le passage des occurrences au type a été étudié dans le champ de la catégorisation, comme dans celui de l'activité ou de la formation professionnelle. L'étude psychologique de la catégorisation conduit - comme on peut s'y attendre - à identifier entre exemplaires et types des relations qui sont parallèles à celles entre mémoire épisodique (les occurrences) et mémoire sémantique (les catégories). Ainsi, Didierjean (2001) montre que l'apprentissage à partir d'exemples met en jeu à la fois la mémoire d'exemplaires et un processus d'abstraction de règles, c'est-à-dire à la fois le positionnement d'un nouveau cas par rapport aux exemples déjà rencontrés, et la recherche ou l'émergence d'invariants (critères, règles) des expériences sédimentées. Des conclusions analogues sont présentées par Erickson et Kruschke (1998).

On peut aussi observer des épisodes « chimères » (composites) : ils se présentent lorsque certains éléments contextuels d'un épisode sont mémorisés comme appartenant à un autre épisode : par exemple on se souvient d'avoir égaré sa voiture dans Marseille, ville qu'on connaît très mal, mais il s'agissait en fait de Toulouse. Différents processus peuvent être à l'origine de ces illusions ${ }^{6}$.

\section{- principes et clinique dans le traitement de cas}

Les relations entre cas et principe définissant une espèce ne sont pas toujours convergentes. Jonsen et Toulmin (2005) discutant de la dialectique « raisonner par principe / évaluer un cas » soulignent la possibilité de discordance entre des décisions consensuelles sur des cas et les divergences exprimées quant aux principes conduisant aux jugements. On peut penser que les processus de décision ne sont pas simplement des processus : «identification de la catégorie, application d'une règle en « si... alors ». De plus, la notion de «principe » en jeu dans des décisions sur des situations complexes fait intervenir des questions d'éthique et de finalité de l'action, voire des dimensions émotionnelles, qui sortent du cadre étroit de l'analyse des processus de catégorisation. Dans de tels domaines, l'apprentissage repose davantage sur l'analyse de la situation dans sa complexité (à base de connaissances) que sur la constitution de catégories de problèmes. Par exemple, dans le domaine médical, après la période sur les recherches sur le diagnostic comme identification du type de cas, des recherches se sont développées sur la place de la « clinique » : l'utilisation de descriptions fines de cas pour la formation est ainsi devenue objet d'étude (par ex. : d'Alessandro, Lewis, \& d'Alessandro, 2004; Wears \& Karsh, 2007).

\section{- place des deux modalités de l'expérience dans l'analogie}

Un exemple représentatif de cette question est une étude de Ball et al. dans le domaine professionnel de la conception (Ball, Ormerod, \& Morley, 2004). Les auteurs analysent le rôle respectif de deux processus d'analogie dans la résolution de problèmes, selon que les concepteurs sont « experts » (longue expérience professionnelle) ou «novices » (en toute fin de formation, avec une expérience acquise en stages).

Le premier processus est dit « orienté schéma » (schema driven). Il consiste en l'application de sché-

6. Ainsi, dans une approche du fonctionnement de la mémoire intégrant approche symbolique et approche connexioniste, Kokinov a montré, d'une part, que l'amalgame d'épisodes, avec intrusion de connaissances générales, peut résulter d'une résolution de problème par analogie (Kokinov \& Zareva-Toncheva, 2001) et, d'autre part, que l'analogie peut produire des effets de « fausse mémoire » pour les deux situations impliquées Kokinov, Feldman, \& Petkov, 2009). La notion d'amalgame considérée dans ces études expérimentales semble en fait voisine de ce que nous avons appelé « chimère ». 
mas de connaissances, intégrant une compréhension abstraite conceptuelle de la nature sous-jacente au problème; ces schémas sont des ressources pour reconnaître des types de problèmes et leur associer des modes de résolution. De tels schémas sont induits par l' " expérience extensive » produite par le traitement d'un grand nombre de problèmes. Le second processus d'analogie est dit « orienté cas » (case driven). Dans ce processus, les sujets cherchent à trouver un problème source analogue antérieurement résolu, qui présente des similarités de surface et dont la solution présente des propriétés qui peuvent être appliquées au problème cible. Les auteurs montrent que 1) les «experts » utilisent globalement davantage de processus d'analogie que les «novices » et 2) que ces processus sont de manière dominante « orientés schèmes » chez les experts, alors qu'ils sont plus souvent « orientés cas » chez les novices. On retrouve la position de Rumelhart et Norman (1981) selon laquelle au cours des apprentissages la connaissance est plongée dans des schémas (mais ces auteurs ne s'appuyaient pas alors sur des études du domaine professionnel). De même Visser a souligné que l'activité de conception pouvait s'appuyer sur des épisodes déjà expérimentés (1995), et qu'elle pouvait se déployer dans des voies diverses, utilisant l'analogie (Visser, 2009).

Dans les termes de notre propre analyse des modalités de l'expérience professionnelle, ce sont les expériences « sédimentées » qui sont les ressources du premier processus d'analogie, que nous traduirions alors par «orienté schème », et les expériences « épisodiques » les ressources du second processus, «orienté cas».

En conclusion, les processus de condensation et typification des épisodes, l'existence d'amalgames ou de « chimères » d'épisodes, les dynamiques entre occurrences et types, relèvent d'un mouvement du singulier, hic et nunc, qui est dans l'instant celui de tout vécu, vers une intégration dans le générique, l'abstrait. Ils introduisent un continuum entre expériences épisodiques et expériences sédimentées en ce qui concerne la connaissance des situations traitées. Toutefois, l'accumulation quantitative des expériences singulières avec une multiplicité de répétitions avec variations a d'autres impacts qualitatifs que l'abstraction dans le champ de la connaissance. Nous attachons certains de ces impacts à la « mémoire du corps », dont font partie autant les schèmes d'action que les engrammes émotionnels, ou les transformations physiques et physiologiques liées à la pratique dans la longue durée de la vie professionnelle (que ne peut mimer aucune situation expérimentale). D'autres tiennent à la densité des variables en jeu dans l'action, et au fait que l'action peut s'y adapter sans pour autant que l'acteur ait eu besoin d'en prendre conscience. Ainsi, bien que liées, expériences épisodiques et expériences sédimentées vont constituer des ressources distinctes, et comporter aussi des «pièges »- sinon des « pathologies »- distincts.

\section{3.- Des ressources et des « pièges » distincts}

Dans la formation et le développement des compétences, il convient de souligner que les expériences sédimentées et les expériences épisodiques ne sont pas permutables; si elles sont des liens étroits, elles ont aussi des spécificités et des places propres.

\section{- les modalités d'expérience et les ressources qu'elles produisent}

Un des impacts spécifiques des expériences sédimentées concerne la dimension « incorporée » des compétences : l'expertise nécessite d'avoir fait et refait le geste, de très nombreuses fois; il en est de même pour la prise en compte « holistique » d'une situation, et pour le repérage rapide d'indices critiques. La possibilité de prendre des décisions rapides déclenchées par un processus de reconnaissance a ainsi été relevée chez les experts, non comme un processus de catégorisation selon des cas types, mais comme l'identification directe d'une situation appelant telle décision (recognition-based decision making : Klein, 1989). Le « lissage » de l'activité, où opérations et actions s'enchaînent sans à-coups, le développement de schèmes, l'automatisation de l'action, relèvent également des spécificités des expériences sédimentées.

À partir des expériences sédimentées se forment aussi des « représentations-en-action » : les repré- 
sentations spatiales en sont peut-être un paradigme. De telles représentations peuvent jouer un rôle dans le contrôle de situations (un «calcul » spatial, par exemple); elles « débordent » les représentations « conceptualisées » qui peuvent s'exprimer et se discuter dans la formation théorique comme dans le récit des épisodes.

En revanche, l'épisode peut donner lieu à description, récit, comme entité singulière identifiable sur laquelle on peut à nouveau travailler. Une des ressources propres aux expériences épisodiques est en effet la possibilité de questionner l'interprétation même d'un épisode, au-delà de ce qu'en permettaient les connaissances au moment où il s'est produit. Cela suppose qu'un niveau suffisant de détail reste «mobilisable » dans les processus de mémorisation.

\section{- biais et limites des deux modalités de l'expérience}

Qu'il s'agisse d'expériences sédimentées ou épisodiques, il est toujours nécessaire d'examiner la nature et la qualité de ce que le sujet acquiert ainsi par expérience : cela dépend très directement du contenu et des conditions de l'expérience. Ce qui est acquis par l'expérience ne dépend pas seulement de la qualité de ce sur quoi elle porte, mais aussi de la manière dont le sujet la perçoit et l'exploite. Les modèles de situations et d'action empiriquement élaborés et ajustés ne permettront pas toujours de traiter tous les cas auxquels pensait le concepteur du travail. On parle souvent dans ces circonstances de schèmes imparfaits, au champ de validité étroit ou déformé par rapport à la compétence visée. Les acquisitions de la compétence par l'expérience seront d'autant plus limitées que la variabilité des situations auxquelles auront à répondre les apprenants sera plus étroite. On assiste alors à une fermeture de la compétence sur elle-même. Elle pourra devenir maximale dans ce champ étroit et très limitée à ce champ. On a là une source de la routinisation de l'activité laquelle sera d'autant plus accentuée que la tâche est elle-même plus élémentaire. Ce phénomène a été souvent décrit comme une conséquence possible d'une automatisation qui ne s'intègre pas à une acquisition plus large (Leplat, 2008b, chap. 2).

Des insuffisances importantes des acquisitions par l'expérience ont été formulées par un chercheur suédois Brehmer (1980) dans un article très critique, que l'on peut relire comme portant sur l'une et l'autre des modalités que nous avons présentées. Ces analyses de Brehmer s'inscrivent dans un courant de recherche qui a été surtout illustré par les travaux de Kahneman et al. consacrés aux jugements dans l'incertitude (Kahneman, Slovik, \& Tverski, 1982). Ce sont, par exemple, les jugements qui peuvent être énoncés dans les situations de risque, du type « je pense que .. », « il est probable que... », « je crains que... ». Ces jugements, souvent fondés sur les expériences, sont entachés de biais que les auteurs précédents ont été inventoriés et décrits. Ces jugements peuvent conduire à des biais, qui entrent dans trois grandes catégories (on en trouve un commentaire dans Leplat, 1985, p. 130 sq.).

- La représentativité. Ce biais favorise l'attribution d'un objet ou d'un processus à une classe dont les membres ont une ressemblance avec l'objet en question. On attribuera par exemple, un incident à une défaillance d'un élément A parce qu'il ressemble à des incidents antérieurs provenant de cette source.

- La disponibilité. Ce biais favorise l'attribution d'un objet ou d'un processus à une catégorie dont les éléments sont plus spontanément évoqués. Par exemple, on attribuera une panne à une défaillance dont on a vu récemment un cas.

- La surconfiance ou conservatisme. Ce biais exprime la tendance à conserver, voire à renforcer un jugement qui a été une fois émis, en dépit de modifications de la situation.

Les modalités de l'expérience peuvent produire des biais de sens inverse (et éventuellement permettre un meilleur équilibre de l'évaluation d'une situation).

Les expériences sédimentées conduisent à une adaptation à la fréquence des situations : les situations rares y sont donc sous-représentées et moins disponibles. Du point de vue des représentations, les expériences réitérées conduisent par « sédimentation » à la construction d'une représentation com- 
mune sous forme d'image opérative. Ces représentations opératives peuvent conduire à une sélection d'indicateurs de situation qui ne sont plus pertinents du fait d'un changement de buts, d'une situation exceptionnelle ou de changements technologiques (des instruments de prise d'information sur la situation ou de l'automatisation de tout ou partie des opérations).

En revanche, les expériences épisodiques vont offrir une disponibilité de situations rencontrées - ou rapportées - et mémorisées justement parce que rares, « hors norme ». Le risque qui a été une fois réalisé devient ainsi un risque pris en compte. Mais cela peut conduire au piège inverse : un épisode marquant peut être faussement pris comme paradigmatique. L'arbre cachant la forêt.

\section{6.- Conclusion}

On a retenu le point de vue de l'expérience comme produit des pratiques dans un domaine et défendu l'idée qu'elle se réalise sous deux modalités : les expériences sédimentées et les expériences épisodiques. Par défaut, le terme d' "expérience », et en particulier d'expérience professionnelle, renvoie dans la littérature à la première modalité. Cependant, la seconde modalité est devenue également un objet d'études dans le champ du travail et de la formation professionnelle.

Concernant les expériences sédimentées, nous avons rappelé trois points majeurs : 1) le rôle de la répétition; 2) l'importance de la variabilité des situations et des perturbations dans la construction des compétences; et enfin 3) l'intériorisation d'un contexte non explicité, qui contribue à la difficulté de partage de ce mode d'expérience.

La modalité des expériences épisodiques renvoie, quant à elle, à une diversité de types de situations considérées dans leur singularité, qui vont de l'exception (qui, on le sait, « confirme la règle » et tout autant l'infirme) au paradigme (le « cas d'école »). Le point commun est le fait qu'il s'agit d'épisodes mémorisés dans leur singularité (et donc conscients) qui, par ailleurs, peuvent être rapportés dans des récits. Leur analyse rejoint les analyses effectuées sur la problématique des « cas » dans la constitution d'un savoir professionnel (spécialement en sciences humaines et sociales).

On peut considérer les expériences sédimentées et les expériences épisodiques comme renvoyant, pour les premières, à la « continuité » du sujet dans le cours de son activité sur le long terme et pour les secondes à la dimension « discrète » des événements singuliers auxquels il a été confronté et qu'il a inscrits en mémoire. Le développement professionnel se nourrit de manière différente de ces deux modalités de l'expérience. En particulier, le développement des schèmes et la dimension « incorporée » des compétences (Leplat, 1995) relèvent étroitement de la qualité et la quantité des expériences sédimentées, cependant que les expériences épisodiques permettent la reprise consciente de situations singulières pour les analyser et/ou les partager avec d'autres acteurs.

Il ne s'agit pas pour autant de modalités étrangères l'une à l'autre. Elles sont articulées sur au moins deux plans : 1) sur le plan de l'organisation et du fonctionnement de la mémorisation par les relations entre mémoire épisodique et mémoire sémantique, avec la modalité «mémoire d'essence » qui fait un pont entre elles; 2) sur le plan de la dynamique entre « cas » (singulier) et « espèce » (générique) pour le traitement de nouvelles situations. Parmi les processus qui mettent en relation ces deux modalités, on peut souligner, d'une part, l'émergence d'invariants opératoires (concepts et schèmes) à partir des expériences sédimentées et, d'autre part, le «mixage » d'épisodes donnant lieu à des sortes d' "épisodes-types », partiellement détachés de leurs contextes particuliers et qui en expriment l'essence (gist).

Nous avons souligné au long de cet article le rôle producteur de l'expérience, dans ses deux modalités. Toutefois, nous avons souligné aussi que l'expérience ne conduit pas de manière inéluctable au développement des compétences. Tout d'abord, nous avons relevé que l'expérience, dans chacune de ses modalités, présente ses propres «pathologies » (limites, dysfonctionnements, biais), dont il faut tenir compte pour analyser ou produire des compétences à partir de l'expérience pour en éviter 
les possibles «effets réducteurs ». Souvent, d'autres conditions que la «qualité » des successions de situations rencontrées doivent être remplies pour assurer les « effets producteurs » de l'expérience : l'expérience doit être outillée, le récit d'épisodes travaillé. À propos des expériences sédimentées, nous avons souligné la nécessité de connaissance du résultat de l'action comme l'une de ces conditions, et plus encore la connaissance de la relation entre l'action et son résultat. Une condition d'un autre type a été soulignée pour l'impact des expériences épisodiques : élever le niveau d'analyse des épisodes, en remontant aux causes dans leur principe et non dans leur réalisation particulière, permet d'en tirer des leçons plus générales.

Une autre condition de productivité, valable pour les deux modalités de l'expérience, est son intégration dans une activité constructive du sujet. La notion de «deliberate practice » utilisée à propos de l'expertise aux échecs (Charness et al., 2005 ; Ericsson, 2004; Ericsson, Krampe, \& Tesch-Römer, 1993) nous semble être une expression - dans un autre cadre théorique - de la place de l'activité constructive pour que les expériences du sujet (sédimentées comme épisodiques) produisent un développement de ses compétences.

La médiation d'autrui pour " outiller » l'expérience est un instrument de l'activité constructive au travail. Une des formes d'aide au développement des compétences y est en effet de nature sociale, par le rôle des pairs et de la hiérarchie de proximité (fonctionnelle) pour favoriser aussi bien des opportunités de situations que la réflexion sur l'action passée.

Si la «qualité » des situations auxquelles on confronte le sujet en formation est une question centrale pour la didactique professionnelle, celle de «l'outillage » de la réflexion sur l'expérience est également reconnue depuis longtemps comme cruciale. Par exemple, dans un ouvrage sur le passage de l'expérience à l'apprentissage, Boud et al. ont justement centré leur discussion sur la place de la réflexion (Boud, Keogh, \& Walker, 1994/1985). Les débriefings ont été mis en avant comme outils de formation (Duke, 1977). Pastré a beaucoup insisté sur l'importance de l'activité des formateurs dans ces débriefings, pour étayer la réflexion sur l'action conduite en situation en centrant la réflexion sur les moments critiques du point de vue de la conceptualisation (Pastré, 2005). Différents travaux ont cependant mis en avant, dans le cas d'élèves ou d'étudiants, le fait qu'un travail réflexif ne pouvait pas toujours être effectué au moment où l'apprentissage s'engageait, qu'un amorçage cognitif était préalable au travail métacognitif.

\section{RÉFÉRENCES}

Anderson, J.R. (Ed.) (1981). Cognitive skills and their acquisition. Hillsdale, N.J.: Lawrence Erlbaum.

Anderson, J.R. (1982). Acquisition of cognitive skills. Psychological Review, 89(4), 369-406.

Anderson, J.R. (1993). The rules of the mind. Hilldale, N.J.: Erlbaum.

Baerentsen, K.B. (1996). Episodic knowledge in system control. In B. Homquvist, P.B. Andersen, H. Klein, $\&$ R. Posner (Eds.), Signs of work. Semiosis and information processing in organisations (pp. 283-323). Berlin: Waletre de Gruyter.

APA. Critical incident technique (CIT). Bibliography. (Site internet consulté le 26 novembre 2010 : pubs/ databases/psyinfo/cit-full.pdf)

Ball, L.J., Ormerod, T.C., \& Morley, N.J. (2004). Spontaneous analogising in engineering design: a comparative analysis of experts and novices. Design Studies, 25, 495-508.

Barriquault, C. (2005). Représentation d'incidents dans le Retour d'Expérience de la navigation aérienne. Thèse de Doctorat de Psychologie, Université Paris 8.

Barriquault, C., \& Rogalski, J. (2001). Inference making during incident analysis in air traffic management. In K. Schubert \& R. Onken (Eds.), CSAPC'01 (pp. 203-213). Munich: Universität der Bundeswehr München. 
Barriquault, C., \& Rogalski, J. (2003). MESOPot : une méthode de questionnement facilitant l'évocation de scénarios potentiels chez les enquêteurs de la navigation aérienne. Rapport Intermédiaire de contrat CENA/CNRS/Université Paris8.

Bartlett, F. C. (1932). Remembering-A Study in Experimental and Social Psychology. Cambridge: Cambridge University Press.

Beaujouan, J., \& Daniellou, F. (2009). Contribution des récits professionnels au développement des apprentissages. L'expérience. $1^{\text {er }}$ Colloque International de l'association Recherches et Pratique en Didactique professionnelle. Dijon: AgroSup, 2-4 Décembre 2009.

Bichindaritz, I. (1995). Incremental concept learning and case-based reasoning: For a cooperative approach. In I. D. Watson (Ed.), Progress in case-based reasoning (pp. 91-106). Berlin: Springer.

Bichindaritz, I., \& Marling, C. (2008). Case-based reasoning in the health sciences: What is next ? Artificial Intelligence in Medicine, 36, 127-135.

Bisseret, A. (1995). Représentation et décision experte. Psychologie cognitive de la décision chez les aiguilleurs du ciel. Toulouse: Octarès.

Bisseret, A., Sebillotte, S., \& Falzon, P. (1999). Techniques pratiques pour l'étude des activités expertes. Toulouse: Octarès.

Boreham, N,, Samurçay, R., \& Fischer, M. (Eds.) (2002). Work Process Knowledge. London: Taylor \& Francis.

Boud, D., Keogh, R., \& Walker (1994/1985). What is reflection in learning? In D. Boud, R. Keogh, \& D. Walker, (Eds.), Reflection: Turning experience into learning (pp. 7-17). London: Routledge-Falmer.

Bourdieu, P. (1980). Le sens pratique. Paris: Les Éditions de Minuit.

Brainerd, C.J., \& Reyna, V.F. (1990). Gist is the gist: The fuzzy trace theory and new intuitionism. Developmental Review, 10, 3-47.

Brassac, C. (2008). Apprendre pour (et à) capitaliser des connaissances : une étude de cas. Travail et Apprentissages, 2, 9-24.

Brehmer, B. (1980). In one word: Not from experience. Acta Psychologica, 45, 223-241.

Bruner, J. (1991). Car la culture donne forme à l'esprit. Paris: Eschel.

Bruner, J. (1996). L'éducation, entrée dans la culture. Paris: Retz.

Burkhardt, J.-M., \& Détienne, F. (1995). La réutilisation des solutions en conception de programmes informatiques. Psychologie Française, 40, 85-98.

Cellier, J.-M. (1990). L'erreur humaine dans le travail. In J. Leplat \& G. de Terssac (Eds.), Les facteurs humains de la fiabilité dans les systèmes complexes (pp. 193-210). Toulouse: Octarès.

Charness, N., Tuffish, M., Krampe, R., Reingold, E., \& Vysukova, E. (2005). The role of deliberate practice in chess expertise. Applied Cognitive Psychology, 19, 151-165.

Clot, Y. (1999). La fonction psychologique du travail. Paris: PUF.

Clot, Y. (2008). Travail et pouvoir d'agir. Paris: PUF.

Clot, Y., \& Leplat, J. (2005). La méthode clinique en ergonomie et en psychologie du travail. Le Travail Humain, 68(4), 289-316.

Conne, F. (2008). L'expérience comme signe didactique indiciel. Recherches en Didactique des Mathématiques, 28(2), 219-264.

Courtois, B. (Ed.) (1989). Apprendre par l'expérience. Éducation Permanente, 100/101.

Courtois, B., \& Pineau, G. (Eds.) (1991). La formation expérientielle des adultes. Paris: La Documentation Française. 
d'Alessandro, D.M., Lewis, T.E., \& d'Alessandro, M.P. (2004). A pedriatic digital storytelling system ofr third-year medical students: The virtual pediatric patients. BMC Medical Education, 4(10). (doi : 10.1186/1472-6920-4-10).

Darses, F., Détienne, F., \& Visser, W. (2004). Les activités de conception et leur analyse. In P. Falzon (Ed.), Ergonomie (pp. 545-563). Paris: PUF.

Didierjean, A. (2001). Apprendre à partir d'exemples : abstraction de règles et/ou mémoire d'exemplaires ? L'Année Psychologique, 101, 325-348.

Drivdahl, S.B., Zaragoza, M.S., \& Learned, D.M. (2009). The role of emotional elaboration in the creation of false memories. Applied Cognitive Psychology, 23(1), 13-35.

Duke, D.L. (1977). Debriefing: a tool for curriculum research and course improvement. Journal of Curriculum Studies, 9, 157-163.

Durand, M., M., Meuwly-Bonte, \& Roublot, F. (2008). Un programme de technologie de formation centré sur une approche auto-référencée de l'activité. Travail et Apprentissages, 1, 76-91.

Erickson, M., \& Kruschke, J. (1998). Rules and examplars in category learning. Journal of Experimental Learning: General, 127, 107-140.

Erickson, T. (1995). Notes on design practice. Stories and prototypes as catalysts for communication. In: Carroll, J.M. (Ed.), Scenario-based Design. Envisioning Work and Technology in System Development (pp. 37-58). New-York: Wiley \& Sons.

Ericsson, K.A. (2004). Deliberate practice and the acquisition and maintenance of expert performance in medicine and related domains. Academic Medicine, 10, S1-S12.

Ericsson, K.A. (2005). Recent advances in expertise research: A commentary on the contributions to the special issue. Applied Cognitive Psychology, 19, 233-241.

Ericsson, K.A., Krampe, R.T., \& Tesch-Römer, C. (1993). The role of deliberate practice in the acquisition of expert performance. Psychological Review, 100, 363-406.

Ericsson, K. A. \& Lehmann, A. C. (1996). Expert and exceptional expertise: a maximal adaptation to task constraints. Annual Review of Psychology, 47, 273-305.

Falzon, P. (1998). La construction des connaissances en ergonomie. In M.-F. Dessaigne \& I. Gaillard (Eds.), Des évolutions en ergonomie (pp. 51-82). Toulouse: Octarès.

Falzon, P., \& Teiger, C. (1995). Construire l'activité. Performances Humaines \& Techniques, $n^{\circ}$ hors-série (septembre), 34-39.

Fischer, M. (2002). Work experience as an element of Work Process Knowledge. In N. Boreham, R. Samurçay, \& M. Fischer (Eds.), Work Process Knowledge (pp. 119-133). London: Taylor \& Francis.

Fischer, M. (2005). The integration of Work Process Knowledge into human resources development. Human Factors and Ergonomics in Manufacturing, 15(4), 369-384.

Fischer, M., Boreham, N., \& Nyhan, B. (Eds.) (2004). European perspectives on learning at work: the acquisition of work process knowledge. Luxembourg: Office for Official Publications of the European Communities.

Fischer, M., \& Rauner, F. (2002). The implications of process knowledge for vocational training. In N. Boreham, R. Samurçay, \& M. Fischer (Eds.), Work Process Knowledge (pp. 160-170). London: Routledge.

Flanagan, J.C. (1954). La technique de l'incident critique. I \& II. Revue de Psychologie Appliquée, avril 1954, 165-185 \& juillet 2004, 267-297.

Gendre, F. (1968/1990). Les critères de réussite dans la mécanique. Un essai d'application de la technique des « incidents critiques ». Le Travail Humain, 31, 47-72. (repris dans J. Leplat (Ed.) (1990), L'analyse du travail en psychologie ergonomique. Recueil de textes, T.2 (pp. 27-57). Toulouse: Octarès.)

George, C. (1983). Apprendre par l'action. Paris: PUF. 
Goldenberg, P., \& Mason, J. (2008). Shedding light on and with examples spaces. Educational Studies in Mathematics, 69(2), 183-194.

Goldsmith, M., Koriat, A., \& Pansky, A. (2005) Strategic regulation of grain size in memory reporting over time. Journal of Memory and Language, 52, 505-525.

Gomm, R., Hammerseley, M., \& Foster, P. (Eds.) (2000). Case-study method. Key issues. Key texts. London: Sage Publications.

Granger, G.-G. (1967). Pensée formelle et sciences de l'homme. Paris: Aubier-Montaigne.

Gremier, D.D. (2004). The critical incident technique in service research. Journal of Service Research, 7(1), 65-89.

Gruber, H.E. (1976-77). Créativité et fonction constructive de la répétition. Bulletin de Psychologie, 327, XXX, 235-239.

Guillaume, P. (1947). La formation des habitudes. Paris: PUF.

Hayes, J.R. (1985). Three problems in teaching general skills. In S.F. Chipman, J.W. Segal, \& R. Glaser (Eds), Thinking and learning skills, vol. 2 (pp. 391-405). Hillsdale, New Jersey: Lawrence Erlbaum.

Hendershot, D .C. (2008). Lessons for industry from the Report of North American Refineries Independent Safety Panel. First Latin American Process Safety Conference and Exhibition, may 27-29, Buenos-Aires, Argentina.

Hoc, J.-M., \& Rogalski, J. (1992). Régulation des activités cognitives et gestion du risque par l'opérateur humain. In P. Dubois \& G. de Terssac (Eds.), Les nouvelles rationalisations de la production (pp. 147168). Toulouse: Cepadues.

Hoffman, R. R., Crandall, B. \& Shadbolt, N. (1998). Use of the Critical Decision Method to Elicit Expert Knowledge: A Case Study in the Methodology of Cognitive Task Analysis. Human Factors, 40(2), 254276.

Hunter, L.P., \& Hunter , L.A. (2006). Storytelling as an educational strategy for midwifery students. Journal of Midwifery \& Women's Health, 51(4), 273-278.

Jonsen, A.R., \& Toulmin, S. (2005). À quoi sert la casuistique? In J.-C. Passeron \& J. Revel (Eds.), Penser par cas (pp. 95-127). Paris: Éditions de l'EHESS.

Kahneman, D., Slovik, P., \& Tversky, A. (Eds.) (1974/1982). Judgment under uncertainty: heuristics and biases. New York: Cambridge University Press.

Karsenty, L. (2001). Méthodes pour la création de mémoires de projet en conception. Revue Française de Gestion Industrielle, 20(1), 35-51.

Kjellen, U. (1987). Deviations and the feedback control of accidents. In J. Rasmussen, K. Duncan, \& J. Leplat (Eds.), New technology and human error (pp. 143-156). Chichester, UK: Wiley.

Klein, G.A. (1989). Recognition-primed decisions. Advances in Man-Machine Systems research, 5, 47-92.

Kokinov, B., Feldman, V., \& Petkov, G. (2009). Analogy-making automatically produces false memories in the both situations. In: B. Kokinov, K. Holyoak, \& D. Gentner (Eds.), New frontiers in analogy research (pp. 249-258). Sofia: NBU Press.

Kokinov, B., \& Zareva-Toncheva, N. (2001). Episode blending as result of analogical problem solving. In Proceedings of the 23rd Annual Conference of the Cognitive Science Society (pp. 510-515). London: Erlbaum.

Leontiev, A. (1972). Le développement du psychisme. Paris: Éditions sociales.

Leplat, J. (1987). Accidents and incidents production: methods of analysis. In J. Rasmussen, K. Duncan, \& J. Leplat (Eds.), New technology and human error (pp. 133-142). Chichester, UK: Wiley.

Leplat, J. (1995). À propos des compétences incorporées. Education Permanente, 123, 101-114.

Leplat, J. (2000). L'analyse psychologique de l'activité en ergonomie. Toulouse: Octarès. 
Leplat, J. (2002). Psychologie de la formation. Jalons et perspectives. Choix de textes (1955-2002). Toulouse: Octarès.

Leplat, J. (2008a). De l'étude de cas à l'analyse de l'activité. In : Repères pour l'analyse de l'activité en ergonomie (pp. 181-226). Paris: PUF. (Antérieurement publié dans Pistes, 2002 ; http://www.pistes.uqam. $\mathrm{ca} / \mathrm{v} 4 \mathrm{n} 2 / \mathrm{articles} / \mathrm{van} 2 \mathrm{a} 8 . \mathrm{htm}$.

Leplat, J. (2008b). Repères pour l'analyse de l'activité en ergonomie. Paris: PUF.

Linhart, R. (1978). L'établi. Paris: Éditions de Minuit.

Marchand, A.-L. (2009). Usages des récits expérientiels et des savoirs épisodiques dans l'apprentissage de la gestion des risques. Thèse d'Ergonomie, CNAM, Paris.

Marchand, A.-L., \& Falzon, P. (2006). Rôle de l'anecdote dans la formation à la gestion du risque. In J. Maline \& M. Pottier (Eds.), Congrès SELF 2006 Ergonomie et société au travail : transformations du travail et perspectives pluridisciplinaires (pp. 579-587). http://www.ergonomie-self.org/content/ content30156.

Mayen, P. (2008). L’expérience dans les activités de Validation des Acquis de l'Expérience. Travail et Apprentissages, 1, 58-75.

Mayen, P., \& Mayeux, C. (2003). Expérience et formation. Savoir, 1, 15-56.

Merleau-Ponty, M. (1945). Phénoménologie de la perception. Paris: Gallimard.

Negny, S., \& Le Lann, J.-M. (2008). Case-based reasoning for chemical engineering design. Chemical engineering research and design, 86, 648-658.

Newell, A., \& Rosenbloom, P.S. (1981). Mechanisms of skill acquisition and law of practice. In J.R. Anderson (Ed.), Cognitive skills and their acquisition (pp. 1-56). Hillsdale, New Jersey: Lawrence Erlbaum.

Norman, D.A. (1993). Things that make us smart. Reading, MA: Addison Wesley.

Norros, L. (2004). Acting under uncertainty. The core-task analysis in ecological study of work. Espoo, Finland: VTT Publications.

O'Hare, D., \& Wiggins, M. (2004). Remembrance of cases past: who remembers what, when confronting critical flight events. Human Factors, 46(2), 277-287.

O’Hare, D., Mullen, N., Wiggins, M., \& Molesworth, B. (2008). Finding the right case: The role of predictive features in memory for aviation accidents. Applied Cognitive Psychology, 22, 1163-1180.

Ochanine, D. (1978). Le rôle des images opératives dans la régulation des activités de travail. Psychologie et Éducation, 2, 63-72.

Olson, S.E., \& Rasmussen, J. (1989). The reflective expert and the prenovice. In L. Bainbridge \& R. Quintanilla,(Eds), Developping skills with information technology (pp. 9-33). London: Wiley.

Passeron, J.-C., \& Revel, J. (Eds.) (2005). Penser par cas. Paris: Éditions de l’EHESS.

Pastré, P. (1997). La didactique professionnelle et le développement. Psychologie Française, 89-100.

Pastré, P. (2005). Apprendre par la résolution de problèmes : le rôle de la simulation. In P. Pastré (Ed.). Apprendre par la simulation. De l'analyse du travail aux apprentissages professionnels (pp. 17-40). Toulouse: Octarès.

Piaget, J., \& Inhelder, B. (1966/2004). La psychologie de l'enfant. Paris: PUF.

Plaza, D. (2009). La gestion des émotions dans un métier de relation de service. Les Animateurs Agents Mobiles engagés dans une Nouvelle Relation de Service. Thèse de Psychologie, Université Paris8.

Rasmussen, J., Pejtersen, A.M., \& Goodstein, L.P. (1994). Cognitive systems engineering. New York: J. Wiley \& Sons.

Richard, J.-F. (2004). Les activités mentales. De l'interprétation de l'information à l'action. Paris: PUF. 
Rikers, R.M.J., \& Paas, F. (2005). Recent advances in expertise research. Applied Cognitive Psychology, 19, 145-149.

Rogalski, J. (2004). La didactique professionnelle : une alternative aux approches de « cognition située » et « cognitiviste » en psychologie des acquisitions. @ctivités, 1(2), 103-120. En ligne : http://www.activites. org/v1n2/Rogalski.pdf

Rogalski, J. (2007). Situations et schèmes. Action et connaissance. In M. Merri (Ed.), Activité humaine et conceptualisation. Questions à Gérard Vergnaud (pp. 161-178). Toulouse: Presses Universitaires du Mirail.

Rogalski, J., \& Marquié, J.-C. (2004). Évolution des compétences et des performances. In J.-M. Hoc \& F. Darses (Eds.), Psychologie ergonomique : tendances actuelles (pp. 142-173). Paris: PUF.

Rooksby, J., Gerry, B., \& Smith, A.F. (2007). Incident reporting schmes and the need for a good story. International Journal of Medical Informatics, 76(supp. 1), 205-211.

Rouve, M.-E., \& Ria, L. (2008). Analyse de l'activité professionnelle d'enseignants néo-titulaires en réseau « ambition réussite » : étude de cas. Travail et Formation en Éducation (http://tfe.revues.org/index565. html - consulté le 28/09/09).

Rumelhart, D.E., \& Norman, D.A. (1981). Analogical processes in learning. In J. Anderson (Ed.), Cognitive skills and their acquisition (pp. 335-359). Hillsdale, NJ: LEA.

Salas, E., \& Rosen, (2010). Experts at work : principles for developing expertise in organisations. In S.W.J., \& Salas, E. (Eds.), Learning, training, and development in organizations (pp. 101-118). New York: Routledge.

Shiffrin, R.M., \& Schneider, W. (1977). Controlled and automatic human information processing, II : Perceptual learning, automatic attending, and a general theory. Psychological Review, 84, 127-190.

Smith, J.A., Harré, R., \& Van Langenhove, L. (1995). Idiography and the case-study. In J.A. Smith, R. Harré \& L. Van Langenhove (Eds.), Rethinking psychology (pp. 59-69). London: Sage Publications.

Theureau, J. (2006). Le cours d'action : méthode développée. Toulouse: Octarès.

Van Gog, T., Paas, F., \& Van Merriëndoer, J.J.G. (2005). Uncovering expertise-related differences in troubleshooting performance: combining eye movement and concurrent verbal protocol data. Applied Cognitive Psychology, 19, 205-221.

Vergnaud, G. (1990). La théorie des champs conceptuels. Recherches en Didactique des mathématiques, 10 $(2 / 3), 133-170$.

Vergnaud, G. (2001-2002). Piaget revisité par la didactique. Intellectica, 33, 107-123.

Vergnaud, G. (2007). Héritages. In M. Merri (Ed.), Activités humaines et conceptualisation. Questions à Gérard Vergnaud (pp. 27-37). Toulouse: Presses Universitaires du Mirail.

Vermersch, P. (1990). Questionner l'action : l'entretien d'explicitation. Psychologie Française, 35, $227-235$.

Vermersch, P., \& Maurel, M. (Eds.) (1997). Pratiques de l'entretien d'explicitation. Paris: ESF.

Vermersch, P. (2000). Approche du singulier. In Centre de Recherches sur la Formation du CNAM (collectif), L'analyse de la singularité de l'action. Paris: PUF.

Visser,W. (1995). Use of episodic knowledge and information in design problem solving. Design Studies, 16(2), 171-187. Also in N. Cross, H. Christiaans, \& K. Dorst (Eds.) (1996). Analysing design activity (Ch. 13, pp. 271-289). Chichester, England: Wiley.

Visser, W. (2009). Design: one, but in different forms. Design Studies, 30(3), 187-223.

Vygotsky, L. (1997/1934)). Pensée et langage. Paris: La dispute.

Wears, R.L., Karsh, B. T. (2007). Thick versus thin: Description versus classification in learning from case reviews. Annals of Emergency Medicine (doi 10.1010). 
Weill-Fassina, A., \& Pastré, P. (2004). Les compétences professionnelles et le développement. In P. Falzon (Ed.), Ergonomie (pp. 213-231). Paris: PUF.

Widlöcher, (1999). La psychologie clinique et pathologique. Psychologie Française, 44(3), 227-232.

Wiggins, M., \& O'Hare, D. (2003). Weatherwise: Evaluation of a cue-based training approach for the recognition of deteriorating weather conditions during flight. Human Factors, 45(2), 337-345.

Woods, D.D. (2006). Essential characteristics of résilience. In E. Hollnagel, D.D. Woods, \& N. Leveson (Eds.), Resilience Engineering. Concepts and precepts (pp. 21-34). Aldershot, Hampshire: Ashgate.

\section{RÉSUMÉ}

On définit ici l'expérience professionnelle comme ce qui est produit sur l'homme par sa pratique, c'est-à-dire par la réalisation des tâches d'un domaine, sur un temps relativement long. On défend l'idée qu'elle présente deux modalités : les expériences « sédimentées » sont centrées sur la répétition des tâches dans leur variabilité ; les expériences «épisodiques » sur la singularité des situations rencontrées, des cas hors norme aux cas paradigmatiques. On discute l'articulation de ces deux modalités, selon les exigences différentes des types de tâches dans les domaines de travail. L'analyse de l'expérience selon ces modalités « sédimentées » et « épisodiques » articulées sert à questionner leurs apports à l'évolution des compétences, à discuter des « pathologies » possibles de l'expérience, à expliciter des conditions nécessaires aux acquisitions et à tirer quelques conséquences sur la conception de formations ${ }^{7}$.

\section{MotS-CLÉS}

Expérience professionnelle, expériences sédimentées, expériences épisodiques, formation et développement des compétences

\section{RÉFÉRENCEMENT}

Rogalski, J., \& Leplat, J. (2011). L'expérience professionnelle : expériences sédimentées et expériences épisodiques. Activités, 8(2), pp. 4-31, http://www.activites.org/v8n2/v8n2.pdf

Article soumis le 6 avril 2010, accepté pour publication le 10 mai 2011

7. Ce texte est issu d'une communication - reprise et modifiée - publiée dans les actes informatiques du premier colloque francophone de Didactique Professionnelle sur l'expérience, Dijon, 1-4 décembre 2010. 